\title{
MODELING TEMPERATURE DISTRIBUTION INSIDE AN EMULSION FUEL DROPLET UNDER CONVECTIVE HEATING: A KEY TO PREDICTING MICROEXPLOSION AND PUFFING
}

\author{
J. Shinjo, ${ }^{1, *}$ J. Xia ${ }^{1}$ A. Megaritis, ${ }^{1}$ L. C. Ganippa, ${ }^{1} \mathcal{E}$ \\ R.F. Cracknell ${ }^{2}$ \\ ${ }^{1}$ Department of Mechanical, Aerospace and Civil Engineering, and \\ Institute of Energy Futures, Brunel University London, Uxbridge UB8 \\ 3PH, United Kingdom \\ ${ }^{2}$ Shell Global Solutions, Shell Technology Centre Thornton, P.O. Box 1, \\ Chester CH1 3SH, United Kingdom
}

*Address all correspondence to: J. Shinjo, E-mail: junji.shinjo@brunel.ac.uk

Original Manuscript Submitted: 01/18/2015; Final Draft Received: 05/17/2015

\begin{abstract}
Microexplosion/puffing is rapid disintegration of a water-in-oil emulsion droplet caused by explosive boiling of embedded superheated water sub-droplets. To predict microexplosion/puffing, modeling the temperature distribution inside an emulsion droplet under convective heating is a prerequisite, since the temperature field determines the location of nucleation (vapor bubble initiation from superheated water). In the first part of the present study, convective heating of water-in-oil emulsion droplets under typical combustor conditions is investigated using high-fidelity simulation in order to accurately model inner-droplet temperature distribution. The shear force due to the ambient air flow induces internal circulation inside a droplet. It has been found that for droplets under investigation in the present study, the liquid Peclet number $P e_{L}$ is in a transitional regime of $100<P e_{L}<500$. The temperature field is therefore somewhat distorted by the velocity field, but the distortion is not strong enough to form Hill's vortex for the temperature field. In the second part of the present study, a novel approach is proposed to model the temperature field distortion by introducing angular dependency of the thermal conductivity and eccentricity of the temperature field. The model can reproduce the main features of the temperature field inside an emulsion droplet, and can be used to predict the nucleation location, which is a key initial condition of microexplosion/puffing.
\end{abstract}

KEY WORDS: emulsion droplet, convective heating, Peclet number, inner-droplet temperature distribution 


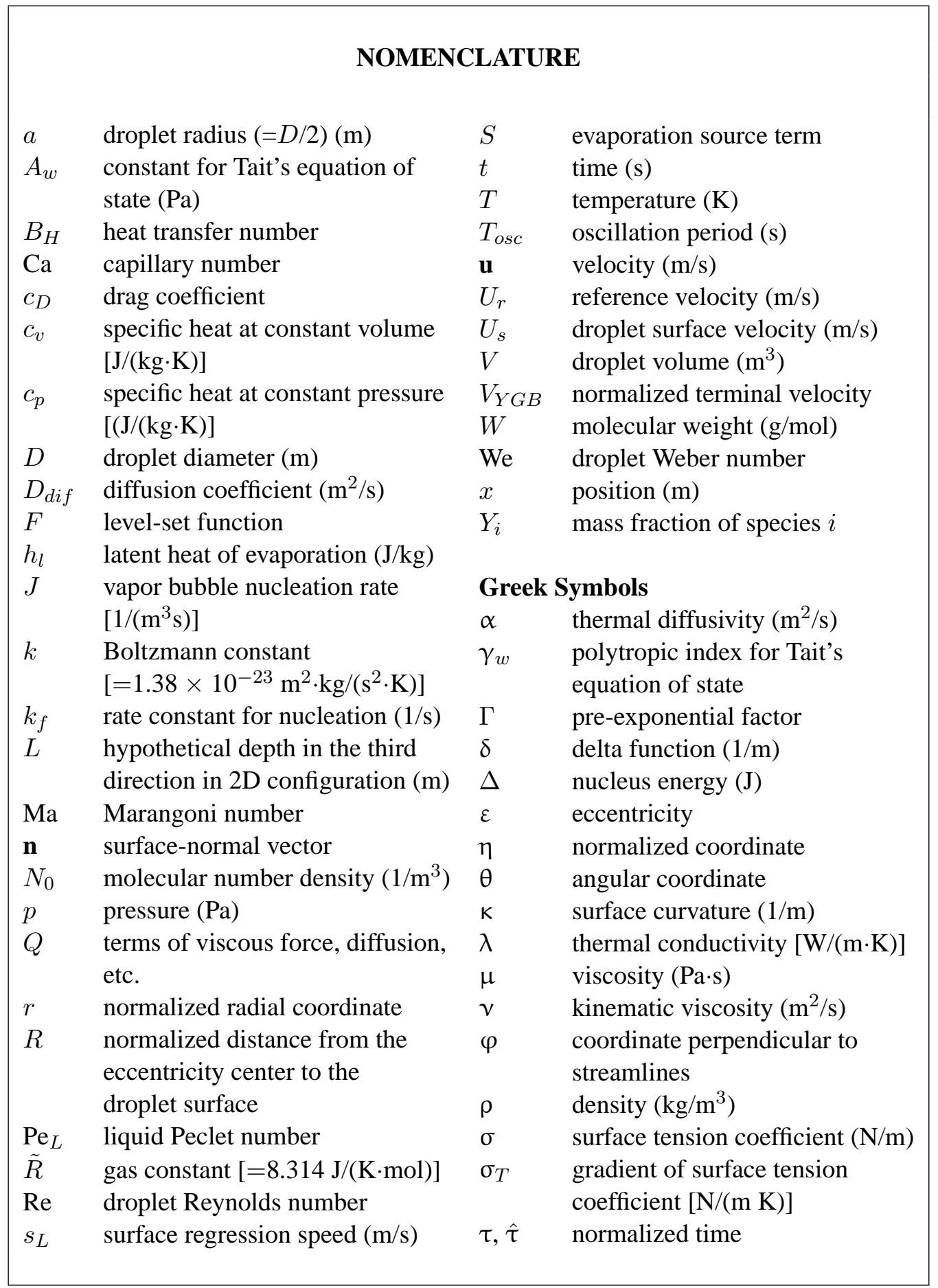




\begin{tabular}{|c|c|c|c|}
\hline \multicolumn{4}{|c|}{ NOMENCLATURE (continued) } \\
\hline \multirow[t]{2}{*}{$x$} & factor for effective thermal & $i$ & inner \\
\hline & conductivity & $i, j$ & indices for sub-droplets \\
\hline \multirow[t]{2}{*}{$\omega$} & angular velocity of oscillation & $L$ & liquid \\
\hline & $(\mathrm{rad} / \mathrm{s})$ & $m$ & modified \\
\hline \multirow[t]{2}{*}{$\dot{\omega}$} & evaporation rate $\left[\mathrm{kg} /\left(\mathrm{m}^{2} \cdot \mathrm{s}\right)\right]$ & $n$ & normal \\
\hline & & $O$ & outer \\
\hline \multicolumn{2}{|c|}{ Subscripts } & $O$ & oil \\
\hline 0 & reference, equilibrium & $p$ & parallel \\
\hline$\infty$ & ambient & parent & parent droplet \\
\hline$a v$ & average & $S$ & surface \\
\hline$b$ & boiling & $V$ & vapor \\
\hline$e f f$ & effective & $W$ & water \\
\hline$e q$ & equivalent & & \\
\hline$G$ & gas & \multicolumn{2}{|c|}{ Superscripts } \\
\hline$H G$ & gas-hydrodynamic & $*$ & normalized time \\
\hline
\end{tabular}

\section{INTRODUCTION}

Spray combustion is widely used in liquid-fueled combustion systems such as combustion engines. Recently, emission regulations for $\mathrm{NO}_{x}, \mathrm{CO}_{2}$, and soot from combustion engines have become more and more stringent due to growing concerns over environmental issues. Using emulsion (emulsified) fuel in combustion engines is considered as one promising solution (Dryer, 1977; Law et al., 1980; Lasheras et al., 1984; Sheng et al., 1994; Segawa et al., 2000; Kadota and Yamasaki, 2002; Fuchihata et al., 2003; Zeng and Lee, 2007; Tarlet et al., 2009; Watanabe et al., 2010; Suzuki et al., 2011; Watanabe and Okazaki, 2013; Shinjo et al., 2014). Emulsion fuel is a blend of immiscible liquids such as oil and water, formed with the help of surfactant agents. Microexplosion/puffing is one of the distinctive features of emulsion droplet dynamics, which can largely enhance secondary atomization and fuel/air mixing.

Microexplosion/puffing is rapid disintegration of an emulsion droplet caused by explosive boiling of embedded water sub-droplets. If the breakup is extensive, it is usually called microexplosion. If the breakup is limited to a portion of the oil droplet, it is called puffing. Inside a combustor, emulsion droplets are heated by the ambient hot air. Since the boiling point of oil is typically higher than that of water, embedded water sub-droplets will be superheated. This state is metastable. With some disturbance, nucleation (vapor bubble formation) occurs and water will start boiling explosively, thus 
causing breakup of the parent oil droplet. If spray conditions are properly controlled toward favorable microexplosion conditions, emulsion fuels will be able to meet conflicting spray requirements of longer penetration depth achieved by large droplets and better evaporation/mixing achieved by small secondary droplets. Another important benefit is that evaporated water reduces the local flame temperature and adds $\mathrm{OH}$ radicals, thereby reducing the emissions of $\mathrm{NO}_{x}$ and soot (Kadota and Yamasaki, 2002).

Accurate prediction of emulsion spray processes under microexplosion/puffing conditions requires knowledge of relevant physical processes such as droplet heating, evaporation, boiling, breakup, and droplet-turbulence interaction. Our current knowledge is still far from sufficient to predict spray dynamics with microexplosion/puffing. Typical engine experiments have been conducted to confirm the overall benefits of emulsion fuels, but the underlying physics remains unknown (Dryer, 1977; Sheng et al., 1994; Kadota and Yamasaki, 2002). In single-droplet experiments, a large droplet of O $(1 \mathrm{~mm})$ was used to enable detailed observation and measurement (Law et al., 1980; Lasheras et al., 1984; Segawa et al., 2000; Kadota and Yamasaki, 2002; Suzuki et al., 2011), but its relevance to fuel spray droplets of $\mathrm{O}(10 \mu \mathrm{m})$ has not been well addressed.

In our previous study (Shinjo et al., 2014), the dynamics of microexplosion/puffing has been clarified by detailed interface-capturing numerical simulation. Explosive boiling of water sub-droplets has been investigated from first principles by resolving all the liquid/gas and liquid/liquid interfaces of an emulsion droplet. Parametric study was performed on the size and nucleation locations of water sub-droplets. New physical insights of microexplosion/puffing have been obtained, including puffing and after-puffing dynamics. Such information is significant to develop a microexplosion/puffing model.

In Shinjo et al. (2014), the initial conditions such as droplet temperature and nucleation locations were prescribed rather than determined. The emulsion droplet temperature was uniform and the initiation of explosive boiling of embedded water subdroplets was triggered by placing a single vapor nucleus at the water/oil interface. For fuel spray droplets, however, these conditions should be determined by the droplet heating process in the convective flow. The initiation of microexplosion/puffing, i.e., the formation of nuclei in superheated water, is strongly dependent on temperature. The homogeneous nucleation theory (Avedisian and Glassman, 1981; Avedisian, 1985) predicts the probability of vapor bubble nucleation rate $J$ as $J=\Gamma k_{f} N_{0} \exp (-\Delta / k T)$, where $\Delta=16 \pi \sigma^{3} / 3\left(p_{V}-p_{L}\right)^{2}$. For a hexadecane/water droplet at $30 \mathrm{~atm}$, the nucleation temperature range is very narrow, i.e., $558-564 \mathrm{~K}$, in which $J\left[1 /\left(\mathrm{m}^{3} \cdot \mathrm{s}\right)\right]$ changes from $J=1$ to $J=10^{10}$. Experiments also confirmed that nucleation occurred within this narrow range of temperature (Avedisian and Glassman, 1981). Therefore, an improved understanding of the temperature distribution inside an emulsion droplet is needed as a key initial condition for accurate modeling of microexplosion/puffing, and will be the aim of this paper. In the literature, the heating process of emulsion droplets has been discussed (Law, 1977, 1982; Law et al., 1980; Sirignano, 1983). However, the knowledge 
is still not sufficient to determine the initial conditions for microexplosion/puffing of emulsion droplets.

The objectives of the current study are to investigate convective heating of an emulsion droplet and to obtain modeling insights into droplet microexplosion/puffing for high-fidelity simulation of fuel spray processes. With the capability of predicting the temperature field inside an emulsion droplet under convective heating, microexplosion/ puffing of emulsion fuel droplets can be properly modeled combined with our previous study (Shinjo et al., 2014). In the present study, the droplet size and flow conditions are set close to those typical in a combustor. The droplet Reynolds number is of $\mathrm{O}(10-100)$ (Sirignano, 2010).

It is known that the surface shear stress induces internal circulation in a liquid droplet (Sirignano, 2010). Law (Law, 1977, 1982; Law et al., 1980) has proposed models at two limits. In the distillation limit where the droplet surface is always covered by a uniform mixture of water and oil due to very strong internal circulation, the surface temperature is bound by the lower boiling point of water. The droplet temperature remains low until water is completely depleted due to evaporation. In this case, microexplosion is unlikely. In contrast, in the frozen mode where there is no internal circulation, the droplet composition remains uniform and equilibrium vaporization is only possible for the abundant species (oil). The attainable droplet temperature is primarily controlled by the boiling point of the oil and microexplosion is possible. Sirignano and coworkers (Prakash and Sirignano, 1978, 1980; Tong and Sirignano, 1982; Abramzon and Sirignano, 1989; Chiang et al., 1992; Sirignano, 2010) have further advanced the discussion on the droplet conditions between the above two extreme limits. For a droplet at a large droplet Reynolds number, the internal circulation is similar to Hill's vortex (ring vortex). This internal circulation can be seen for a range of droplet Reynolds numbers (Dwyer and Sanders, 1984; Talley and Yao, 1986; Dandy and Leal, 1989; Sirignano, 2010; Bergeles et al., 2014). Whether the temperature field follows the velocity field is controlled by the liquid Peclet number $\mathrm{Pe}_{L}=D U_{s} / \alpha$. This is the ratio of the advective heat transport rate (in the direction along vortex streamlines) to the diffusive heat transport rate (in the direction normal to vortex streamlines). If $\mathrm{Pe}_{L}$ is large $\left(\mathrm{Pe}_{L}>1000\right.$, Abramzon and Sirignano, 1989), the distribution of the temperature field becomes similar to the shape of Hill's vortex. If $\mathrm{Pe}_{L}$ is small ( $\mathrm{Pe}_{L}<100$, Abramzon and Sirignano, 1989), the inner-temperature distribution is not strongly affected by the internal circulation. For emulsion droplets of $\mathrm{O}(10 \mu \mathrm{m})$ under investigation in the present study, it will be shown that $\mathrm{Pe}_{L}$ lies between the above two limits and is in a transitional regime, i.e., $100<\mathrm{Pe}_{L}<500$. A new model is therefore needed to predict the inner-droplet temperature distribution under such a regime. Compared to a model for mono-component droplets, the new model may also need to take into consideration the effects of water sub-droplets on the temperature field in an emulsion droplet.

It has been reported that aggregation and coalescence of dispersed water sub-droplets may occur when the inner-droplet temperature is high and stratified (Segawa et al., 2000;

Volume 26, Issue 6, 2016 
Kadota and Yamasaki, 2002; Suzuki et al., 2011). Aggregation of sub-droplets is due to thermocapillary (Marangoni) migration and coalescence of sub-droplets is due to reduced effectiveness of the surfactants at high temperature (Segawa et al., 2000; Suzuki et al., 2011). In enlarged-scale single-droplet experiments, these phenomena may play a role, but for real-scale small spray droplets of $\mathrm{O}(10 \mu \mathrm{m})$, it is considered that there is no sufficient time for these phenomena to develop. Recently, Watanabe and Okazaki (2013) have found that complete microexplosion rarely occurred and puffing was more likely for smaller spray droplets of $\mathrm{O}(10 \mu \mathrm{m})$. The time from spray injection to puffing was around $0.5 \mathrm{~ms}$ and they argued that water coalescence would not occur extensively in such a short time. Therefore, in this study, thermocapillary migration and coalescence of water sub-droplets are not considered.

In the following, Section 2 describes the mathematical formulations and numerical methods. Section 3 presents two validation cases. Section 4 shows the results of convective heating of emulsion droplets and modeling of inner-droplet temperature distribution based on the insights gained from simulation. Finally in Section 5, concluding remarks are given.

\section{MATHEMATICAL FORMULATIONS AND NUMERICAL METHODS}

\subsection{Governing Equations}

The governing equations are Navier-Stokes equations of density $\rho$, velocity $\mathbf{u}$, temperature $T$, and species mass fraction $Y_{i}$ (Yabe et al., 2001; Shinjo et al., 2014),

$$
\partial \mathbf{f} / \partial t+(\mathbf{u} \cdot \nabla) \mathbf{f}=\mathbf{g},
$$

where

$$
\begin{aligned}
& \mathbf{f}=\left(\rho, \mathbf{u}, T, Y_{i}\right), \\
& \mathbf{g}=\left(-\rho \nabla \cdot \mathbf{u}+S_{\rho},-\frac{\nabla p}{\rho}+\mathbf{Q}_{\mathbf{u}}+\mathbf{S}_{\mathbf{u}},-\frac{P_{T H} \nabla \cdot \mathbf{u}}{\rho c_{v}}+Q_{T}+S_{T}, Q_{Y_{i}}\right) .
\end{aligned}
$$

The general form for $P_{T H}$ is $P_{T H}=T(\partial p / \partial T)_{\rho}$ and for an ideal gas $P_{T H}=p \cdot \mathbf{Q}_{\mathbf{u}}$ includes the viscous and surface tension forces. $Q_{T}$ includes the work done by viscous forces and heat conduction modeled by Fourier's law. $Q_{Y_{i}}$ is the mass diffusion term modeled by Fick's law. Four species of oil, $\mathrm{H}_{2} \mathrm{O}, \mathrm{O}_{2}$, and $\mathrm{N}_{2}$ are considered. The vapor mass fraction at the droplet surface $Y_{V}$ is determined by the Clapeyron-Clausius relation (Tanguy et al., 2007).

$$
\begin{aligned}
& p_{V}=p_{\infty} \exp \left[-\frac{h_{l} W_{V}}{\tilde{R}}\left(\frac{1}{T}-\frac{1}{T_{b}}\right)\right], \\
& Y_{V}=\frac{p_{V} W_{V}}{\left(p_{\infty}-p_{V}\right) W_{G}+p_{V} W_{V}} .
\end{aligned}
$$


$S_{*}$ are the evaporation source terms, given by

$$
S_{\rho}=\rho\left(\rho_{G}^{-1}-\rho_{L}^{-1}\right) \dot{\boldsymbol{\omega}} \delta, \quad \mathbf{S}_{\mathbf{u}}=\left(\rho_{G}^{-1}-\rho_{L}^{-1}\right) \dot{\boldsymbol{\omega}}(\dot{\boldsymbol{\omega}} / \rho) \delta \mathbf{n}, \quad S_{T}=-\left(h_{l} / \rho c_{p}\right) \dot{\boldsymbol{\omega}} \delta .
$$

The evaporation rate at the oil-droplet surface is determined by the jump conditions of heat and mass fractions (Tanguy et al., 2007),

$$
\begin{aligned}
& h_{l} \dot{\boldsymbol{\omega}}=[\lambda \nabla T \cdot \mathbf{n}], \\
& \dot{\omega}\left(Y_{i, G}-Y_{i, L}\right)=\left[\rho D_{d i f} \nabla Y_{i} \cdot \mathbf{n}\right] .
\end{aligned}
$$

The square brackets denote the difference of a variable $f$ between the liquid and gas phases at the interface, i.e., $[f]=f_{L}-f_{G}$.

Interfaces are captured by the level-set method (Sussman et al., 1994; Sussman and Puckett, 2000). Since multiple interfaces are captured in the current study, two level-set functions (signed distance functions) $F_{O}, F_{W}$ are used. $F_{O}=0$ represents the surface of the parent oil droplet, i.e., the interface between the liquid oil and the ambient gas. $F_{W}$ $=0$ represents the surface of embedded water sub-droplets, i.e., between the liquid water and the liquid oil. They both follow, in the general form,

$$
\partial_{t} F+(\mathbf{u} \cdot \nabla) F=-|\nabla F| s_{L},
$$

where $s_{L}=\dot{\omega} / \rho_{L}$ is the surface regression speed due to evaporation. As long as water sub-droplets are embedded in the parent oil droplet, evaporation of water does not occur and the regression speed is zero. An extension of this formulation will be later given in Section 2.3.

Surface tension is modeled by the CSF (continuum surface force) method (Brackbill et al., 1992). With a constant surface tension coefficient $\sigma$, the surface tension force is

$$
\mathbf{F}_{s}=\sigma \kappa \delta \mathbf{n}
$$

This is included in $\mathbf{Q}_{\mathbf{u}}$ as $\mathbf{F}_{s} / \rho$. If $\sigma$ is temperature dependent, $\mathbf{F}_{s}$ is (Herrmann et al., 2008; Ma and Bothe, 2011; Liu et al., 2012)

$$
\mathbf{F}_{s}=\sigma \kappa \delta \mathbf{n}+[\nabla \sigma-(\nabla \sigma \cdot \mathbf{n}) \mathbf{n}] \delta
$$

where the first term on the right-hand side (RHS) is the isothermal part in the surfacenormal direction [see Eq. (8)] and the second term represents the temperature variation effect in the surface-tangential direction. If the surface temperature of a droplet differs locally, the force imbalance induces a flow motion inside (and outside) the droplet (Young et al., 1959; Balasubramaniam and Subramanian, 1996; Hadland et al., 1999; Nas and Tryggvason, 2003; Herrmann et al., 2008; Ma and Bothe, 2011; Liu et al., 2012). In this study, Eq. (8) is used in most cases. In Section 4.3, the thermocapillary effect on the parent droplet is additionally investigated using Eq. (9). 
The equation of state (EOS) for gas is given by the ideal-gas law, and the EOS for liquid water and oil is given by Tait's empirical formulation (Igra and Takayama, 2001),

$$
\frac{p+A_{w}}{p_{0}+A_{w}}=\left(\frac{\rho}{\rho_{0}}\right)^{\gamma_{w}}
$$

where $A_{w}=296.3 \mathrm{MPa}$ and $\gamma_{w}=7.415$.

\subsection{Interface-Capturing Numerical Methods}

The numerical procedure (CIP-CUP method) is based on the CIP (cubic interpolated pseudo-particle or constrained interpolation profile) method (Takewaki et al., 1985),

combined with its extension to both incompressible and compressible flows by the CUP (combined and unified procedure) method (Yabe et al., 2001).

In the CIP-CUP method, Eq. (1) is split into three stages: the advective, nonadvective, and acoustic phases (Shinjo et al., 2014). In the advective phase,

$$
\partial \mathbf{f} / \partial t+(\mathbf{u} \cdot \nabla) \mathbf{f}=0
$$

is solved. The third-order scheme uses a third-order polynomial curve fitting for both flow variables and their derivatives to solve advection (Takewaki et al., 1985; Yabe et al., 2001). In the nonadvective and acoustic phases,

$$
\partial \mathbf{f} / \partial t=\mathbf{g}
$$

is solved by the CUP method (Yabe et al., 2001). This method has been successfully used in our previous studies of turbulent atomization (Shinjo and Umemura, 2010,2011), spray evaporation (Shinjo and Umemura, 2013; Shinjo et al., 2015), and emulsion droplet microexplosion/puffing (Shinjo et al., 2014).

The level-set method is combined with the MARS (multi-interface advection and reconstruction solver) method (Kunugi, 1997), a kind of VOF (volume of fluid) method, to improve mass conservation (Himeno and Watanabe, 2003).

In the standard level-set formulation, droplet coalescence occurs if two droplets contact each other. For an emulsion droplet in the present study, however, an additional numerical treatment is required to prevent such coalescence (see discussions in Section 1). In the next subsection, the extension of the level-set method is explained.

\subsection{Multiple Level-Set Functions for Emulsion (MLE) Method}

Inside an emulsion droplet, coalescence of water sub-droplets is prevented by added surfactants. Physically, the effectiveness of surfactants is dependent on temperature. At low temperature, surfactants can effectively prevent coalescence. At high temperature, 
the ability is weakened, and coalescence of water sub-droplets occurs. Therefore, water sub-droplet coalescence occurs after they have been heated for a certain time in a combustor (Segawa et al., 2000; Suzuki et al., 2011).

In the standard level-set formulation where water sub-droplets are captured by only one single level-set function $F_{W}$, droplet coalescence naturally occurs if the two subdroplets get very close to each other. This is because neighboring droplets can be directly detected through the calculation of $\nabla F_{W}$. Figure 1 shows an example of coalescence of water sub-droplets in a two-dimensional (2D) configuration, using the single level-set function method. The initial flow conditions are $D=30 \mu \mathrm{m}, T_{G}=900 \mathrm{~K}, U_{G}=10 \mathrm{~m} / \mathrm{s}$, $p=10 \mathrm{~atm}, \operatorname{Re}=30$, and $\mathrm{We}=0.58$. In this result, coalescence of water sub-droplets has occurred multiple times, which is physically not correct at low temperature.

This coalescence can be prevented, if multiple level-set functions $F_{W, i}$ are introduced. A schematic of the multiple level-set functions for emulsion (MLE) method is shown in Fig. 2. Figure 2(a) shows the schematic of the standard level-set method where only one level-set function $F_{W}$ is used. When two interfaces get close, they coalesce. Figure 2(b) shows the schematic of MLE, in which, for a droplet of $F_{W, i}$, all the neighboring droplets are expressed by different level-set functions $F_{W, j(j \neq i)}$. In calculating $\nabla F_{W, i}$, all the other level-set functions $F_{W, j(j \neq i)}$ are numerically invisible and therefore their influences are not included. The sub-droplet captured by $F_{W, i}$ behaves as if there are no other sub-droplets in the neighborhood, and therefore coalescence can be prevented. It should be noted that this numerical method does not violate the physics. The neighboring sub-droplets of $F_{W, j(j \neq i)}$ can be implicitly detected through the velocity field and the density difference.
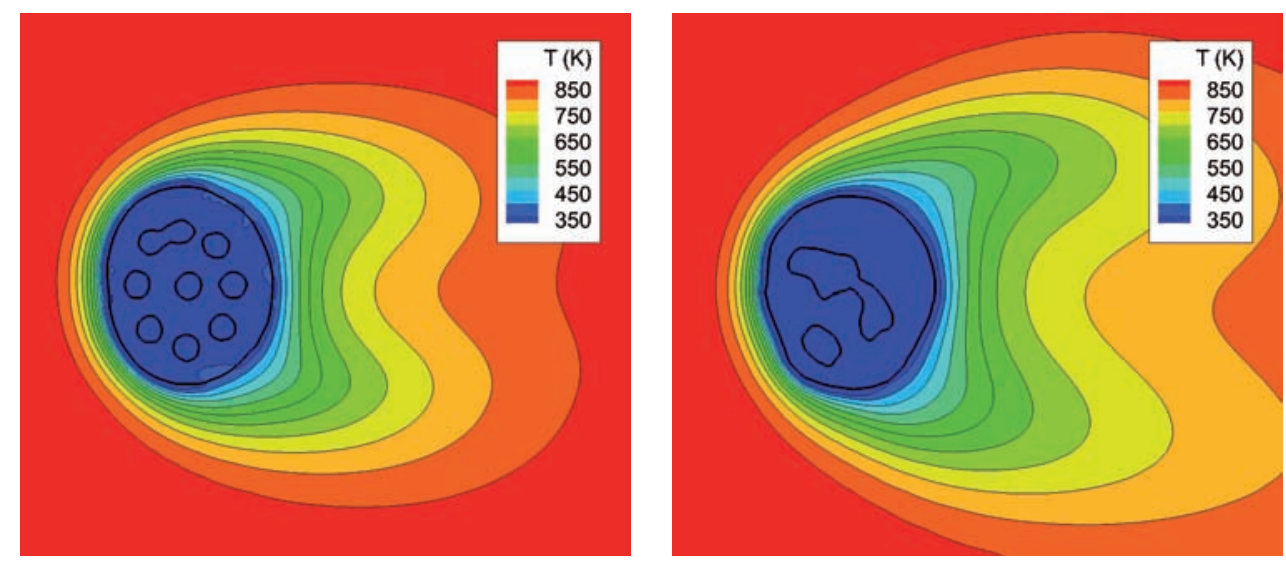

FIG. 1: Unphysical coalescence of water sub-droplets caused by using one single levelset function. $t=10 \mu \mathrm{s}$ (left) and $t=20 \mu \mathrm{s}$ (right). The black solid lines denote the interface shape of the parent oil droplet and water sub-droplets, and the color contours show the temperature field. 


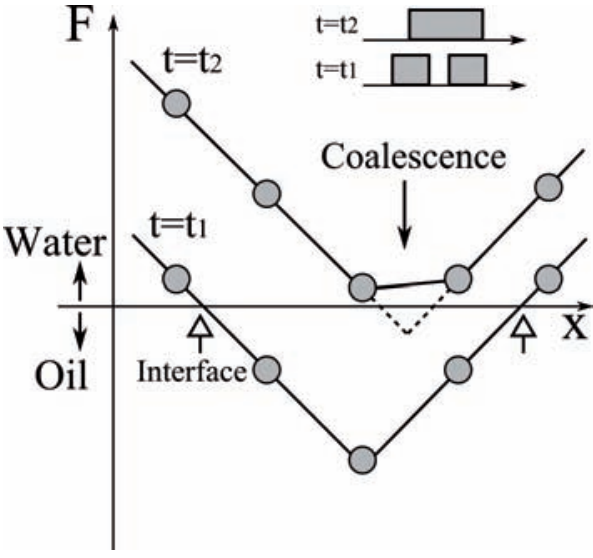

(a)

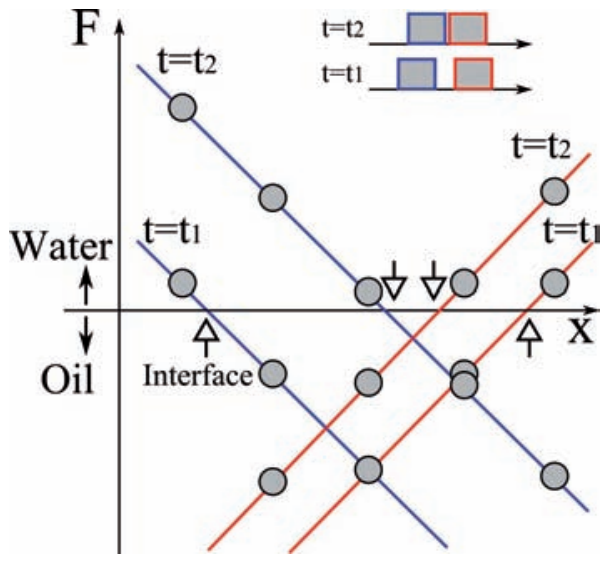

(b)

FIG. 2: Prevention of water sub-droplet coalescence by the MLE method. Arrows with open head indicate the interface locations. In (b), the red and blue lines represent different $F_{W, i}$. (a) standard level-set method; (b) MLE method.

Figure 3 shows the result of the same test case as in Fig. 1, computed with the MLE method. Even after a longer time than that in Fig. 1, unphysical coalescence does not occur and has been prevented. The MLE method only requires that all the neighboring sub-droplets be expressed by different level-set functions. The number of required levelset functions for computation can be made smaller than the actual number of all the sub-droplets by choosing a proper combination of different level-set functions as shown in Fig. 3(a).

\subsection{Case Setup}

In this study, fuel properties of $n$-dodecane are used for the oil. Obtained results can be extended to other typical hydrocarbon fuels that have similar physical properties. The physical properties are taken from the NIST (National Institute of Standards and Technology) database (NIST, 2011). The physical properties for $n$-dodecane at the initial temperature $300 \mathrm{~K}$ are the density of $\rho_{O}=746.5 \mathrm{~kg} / \mathrm{m}^{3}$, heat capacity $c_{p, O}=2.2164$ $\times 10^{3} \mathrm{~J} / \mathrm{kg} / \mathrm{K}$, viscosity $\mu_{O}=1364.2 \times 10^{6} \mathrm{~Pa} \cdot \mathrm{s}$, thermal conductivity $\lambda_{O}=0.13589$ $\mathrm{W} / \mathrm{m} / \mathrm{K}$, latent heat of evaporation $h_{l, O}=256 \times 10^{3} \mathrm{~J} / \mathrm{kg}$, and surface tension $\sigma_{O}=$ $24.8 \times 10^{-3} \mathrm{~N} / \mathrm{m}$. For water, $\rho_{W}=997.85 \mathrm{~kg} / \mathrm{m}^{3}, c_{p, W}=4.1725 \times 10^{3} \mathrm{~J} / \mathrm{kg} / \mathrm{K}, \mu_{W}=$ $853.3 \times 10^{6} \mathrm{~Pa} \cdot \mathrm{s}, \lambda_{W}=0.61163 \mathrm{~W} / \mathrm{m} / \mathrm{K}, h_{l, W}=2257 \times 10^{3} \mathrm{~J} / \mathrm{kg}$, and $\sigma_{W}=70.0 \times$ $10^{-3} \mathrm{~N} / \mathrm{m}$. The temperature dependence of each property is taken into account.

The initial droplet diameter is $D=30 \mu \mathrm{m}$. The ambient gas temperature is $T_{G}=$ $900 \mathrm{~K}$ and the initial droplet temperature is $T_{L}=300 \mathrm{~K}$. The ambient pressure is set at $p=1 \mathrm{MPa}$ and the air velocity $U_{G}=10 \mathrm{~m} / \mathrm{s}$. The droplet Reynolds number, based on the 


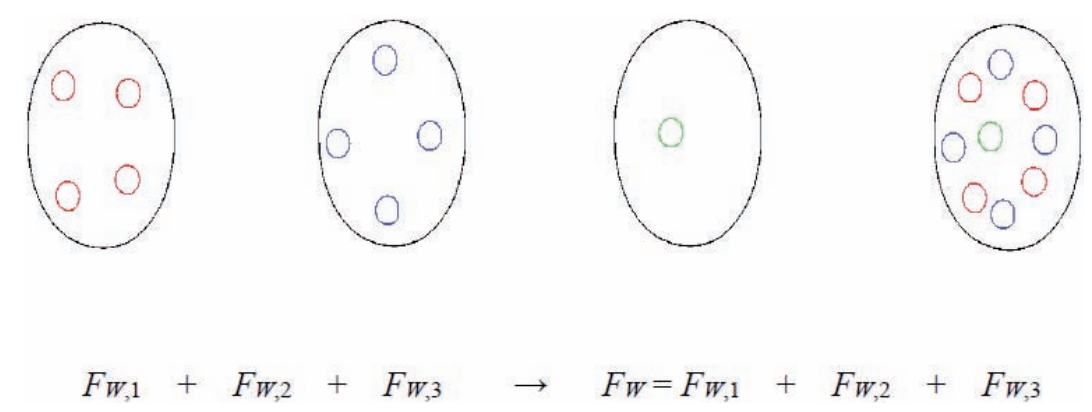

(a)
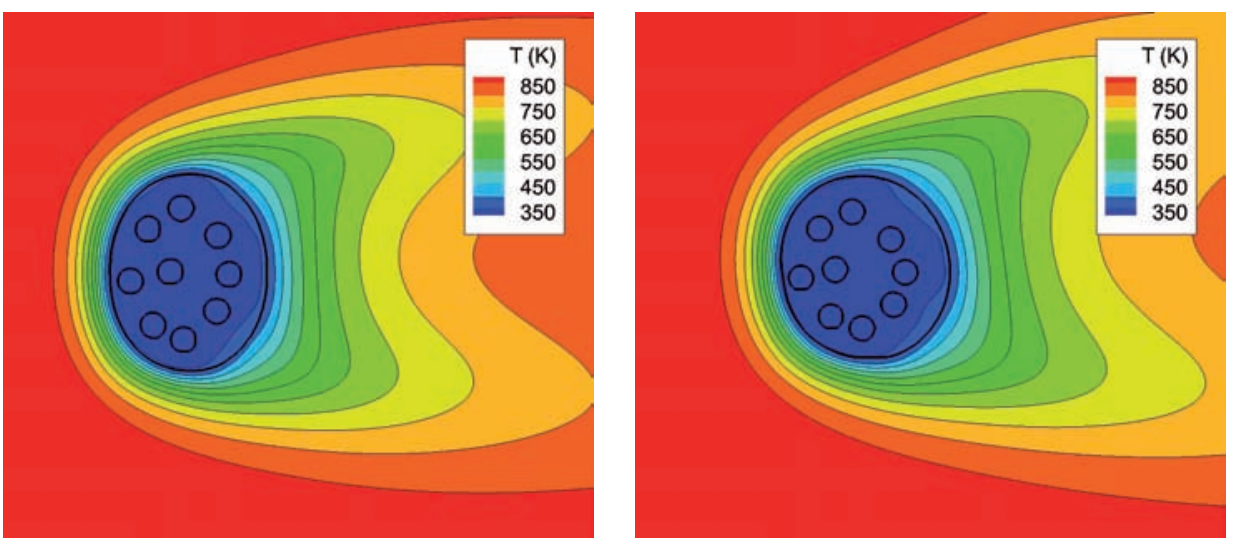

(b)

FIG. 3: Correctly reproduced result of an emulsion droplet by MLE without sub-droplet coalescence. The initial conditions are the same as those of Fig. 1. (a) Schematic of using three level-set functions to express nine water sub-droplets; (b) prevented coalescence of water sub-droplets at $t=20 \mu \mathrm{s}$ (left) and $t=30 \mu$ s (right).

droplet diameter and the relative velocity between the droplet and the gas, is $\operatorname{Re}=30$ and the Weber number is $\mathrm{We}=0.43$. Since the Weber number is small, any further breakup due to aerodynamic force will not occur.

The water sub-droplet size is shown in Table 1 . The water volume fraction is defined as a ratio of the water volume to the parent droplet volume $\left(V_{W} / V_{\text {parent }}\right)$. In threedimensional (3D) cases, it is computationally expensive to treat many water sub-droplets, so the water volume fraction is set relatively smaller. However, the reduction of the water volume fraction due to the consideration of computational cost will not affect results analysis and model development. The number of grid points is $336 \times 336$ for $2 \mathrm{D}$ cases and $336 \times 336 \times 336$ for $3 \mathrm{D}$ cases. Following the conclusion of grid independence in Section 3.1, the grid spacing is set to be $0.35 \mu \mathrm{m}$. The gas velocity is fixed at the inlet boundary and all the other boundaries are open boundaries. 
TABLE 1: Droplet configuration

\begin{tabular}{|c|c|c|c|c|c|c|}
\hline Case & Dimension & $\begin{array}{c}\text { Parent } \\
\text { droplet } \\
\text { diameter } \\
(\mu \mathbf{m})\end{array}$ & $\begin{array}{c}\text { Water } \\
\text { sub-droplet } \\
\text { diameter } \\
(\mu \mathbf{m})\end{array}$ & $\begin{array}{c}\text { Number of } \\
\text { water } \\
\text { sub-droplets }\end{array}$ & $\begin{array}{c}\text { Volume } \\
\text { fraction } \\
\text { of water }\end{array}$ & $\begin{array}{c}\text { Droplet } \\
\text { type }\end{array}$ \\
\hline 1 & 3D & 30 & 2.86 & 19 & $1.6 \%$ & W/O emulsion \\
\hline 2 & 3D & 30 & 4.29 & 19 & $5.6 \%$ & W/O emulsion \\
\hline 3 & 2D & 30 & 2.86 & 9 & $8.2 \%$ & W/O emulsion \\
\hline 4 & 2D & 30 & 4.29 & 9 & $18.4 \%$ & W/O emulsion \\
\hline 5 & 2D & 30 & - & - & - & Oil \\
\hline 6 & 2D & 30 & - & - & - & Water \\
\hline $3 \mathrm{M}$ & 2D & 30 & 2.86 & 9 & $8.2 \%$ & $\begin{array}{c}\text { Case 3 with } \\
\text { Marangoni } \\
\text { effect }\end{array}$ \\
\hline $5 \mathrm{M}$ & 2D & 30 & - & - & - & $\begin{array}{c}\text { Case 5 with } \\
\text { Marangoni } \\
\text { effect }\end{array}$ \\
\hline
\end{tabular}

\section{CODE VALIDATION}

In order to validate the current numerical code, two cases are conducted to examine key phenomena related to convective heating of an emulsion droplet. For other validation cases, satisfactory results have been already obtained. They include linear/nonlinear droplet oscillations (Shinjo et al., 2014), capillary wave dynamics and droplet pinch-off (Shinjo and Umemura, 2010), and boiling surface dynamics (Shinjo et al., 2014).

\subsection{Convective Heating of an Evaporating Mono-component Droplet}

First, convective heating of an evaporating mono-component droplet is considered (Chiang et al., 1992). The initial flow conditions are $D=70 \mu \mathrm{m}, T_{L}=300 \mathrm{~K}, T_{G}=1250 \mathrm{~K}$, $U_{G}=25 \mathrm{~m} / \mathrm{s}, p=10 \mathrm{~atm}$, and $\operatorname{Re}=100$. The flow field is assumed to be axisymmetric, and the droplet deformation is not considered (the shape is always spherical). Two grid systems $(320 \times 82$ and $480 \times 124)$ have been tested and the difference in result is small, so the coarser grid is used here.

The gas flow gradually slows down as time passes due to the drag force on the droplet surface. Accordingly, the Reynolds number Re reduces. Due to the shear force from the air flow, transient development of internal circulation and temperature stratification is observed inside the droplet. Figures 4(a) and 4(b) show the temperature and streamlines at $t_{H G}=5$ where $t_{H G}=4 \mu_{G} t / D^{2} \rho_{G}$. It is clear that the inner-droplet temperature and velocity fields are both similar to Hill's vortex (Sirignano, 2010). The liquid Peclet number $\mathrm{Pe}_{L}$ for this case is on the order of $\mathrm{Pe}_{L} \sim 1000$. Therefore, the similarity between the streamlines and temperature fields can be expected. The droplet surface-averaged 


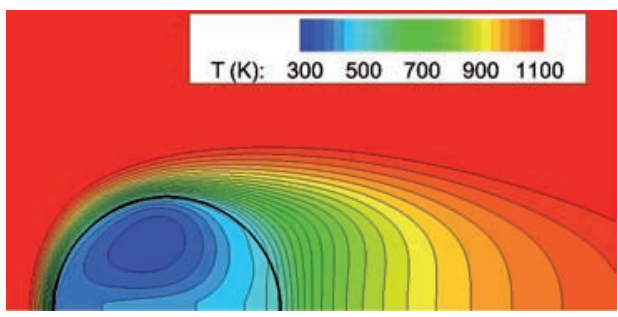

(a)

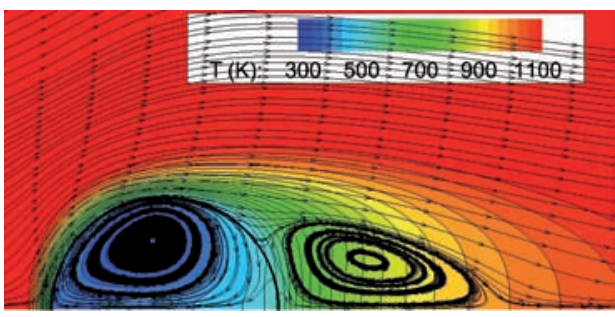

(b)

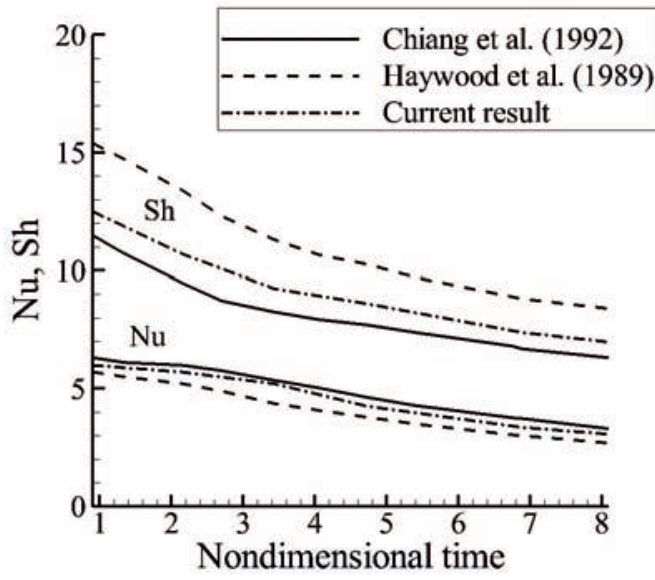

(c)

FIG. 4: Validation on droplet convective heating; (a) temperature field at $t_{H G}=5$, (b) streamlines at $t_{H G}=5$, and (c) Nusselt and Sherwood numbers.

Nusselt and Sherwood numbers, which represent the averaged heat and mass fluxes, are shown in Fig. 4(c). It can be seen that the obtained simulation results are in good agreement with the reported ones (Haywood et al., 1989; Chiang et al., 1992).

\subsection{Thermocapillary (Marangoni) Effect}

When a droplet is placed in a temperature field that has a gradient in one direction, the droplet will move in the temperature gradient direction toward higher temperature (Young et al., 1959; Balasubramaniam and Subramanian, 1996; Hadland et al., 1999; Nas and Tryggvason, 2003; Herrmann et al., 2008; Ma and Bothe, 2011; Liu et al., 2012). This migration occurs due to the difference in local surface tension on the droplet surface [see Eq. (9)]. Due to this force imbalance, flow is induced both inside and outside the droplet, and as a result, the droplet moves toward the higher temperature side, as schematically shown in Fig. 5(a). Although thermocapillary migration of embedded water sub-droplets is considered to be minor in this study (see Section 1), the surface 


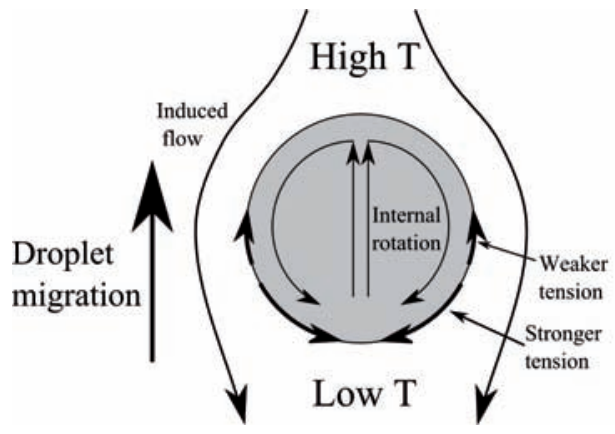

(a)

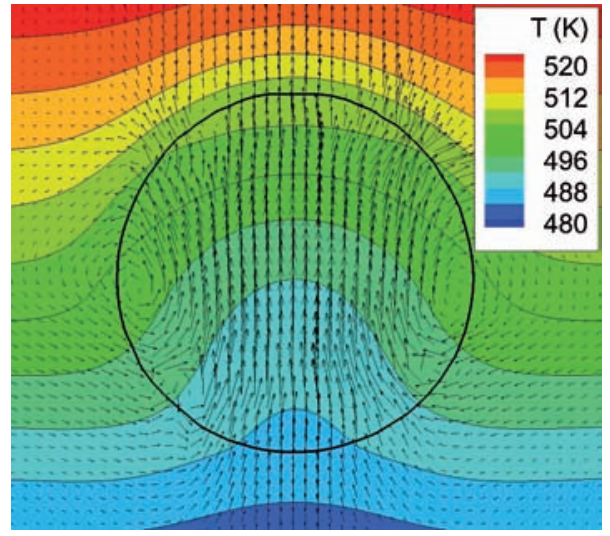

(b)

FIG. 5: Thermocapillary droplet migration for $\mathrm{Ma}=55.5, \mathrm{Re}=3.8$, and $\mathrm{Ca}=0.12$; (a) schematic of flow field, (b) calculated flow field.

tension variation of the parent oil droplet is discussed in Section 4.3. Therefore, code validation is performed here.

Thermocapillary migration of a 3D water droplet bathed in oil is simulated. The embedded water droplet conditions are $D=7.5 \mu \mathrm{m}, \rho_{W}=1000 \mathrm{~kg} / \mathrm{m}^{3}, \mu_{W}=160 \times$ $10^{-6} \mathrm{~Pa} \cdot \mathrm{s}$, and $\lambda_{W}=0.68 \mathrm{~W} / \mathrm{m} / \mathrm{K}$. For the ambient oil, $\rho_{O}=250 \mathrm{~kg} / \mathrm{m}^{3}, \mu_{O}=860 \times$ $10^{-6} \mathrm{~Pa} \cdot \mathrm{s}$, and $\lambda_{O}=0.13 \mathrm{~W} / \mathrm{m} / \mathrm{K}$. To make the calculation easier, the oil density is modulated. The purpose of the modulation is to accelerate, but not to change, the flow dynamics. The surface tension coefficient is $\sigma_{W}(T)=\sigma_{r}\left(1-T / T_{r}\right)^{n}\left[1-m\left(1-T / T_{r}\right)\right]$, where $\sigma_{r}=205.32 \times 10^{-3} \mathrm{~N} / \mathrm{m}, T_{r}=625.7 \mathrm{~K}, n=11 / 9$, and $m=0.6132$ (Vega and de Miguel, 2007). The Reynolds, Marangoni, and capillary numbers are defined as $\mathrm{Re}=$ $U_{r} a / \nu_{O}, \mathrm{Ma}=U_{r} a / \alpha_{O}$, and $C a=U_{r} \mu_{O} / \sigma$, respectively, with the reference velocity $U_{r}=\sigma_{T} a|\nabla T| / \mu_{O} .|\nabla T|$ is the magnitude of temperature gradient and $\sigma_{T}=-d \sigma / d T$ (Young et al., 1959).

Figure 5(b) shows the flow field at $t^{*}=7.8$ (normalized by $a / U_{r}$ ) for $\mathrm{Ma}=55.5$, $\mathrm{Re}=3.8$, and $\mathrm{Ca}=0.12$. The rotational motion inside the droplet can be seen. Initially, the background temperature contour lines are straight and parallel, but they are curved due to the droplet's migration motion. Young et al. (1959) gave the theoretical terminal velocity (normalized by $U_{r}$ ) as

$$
V_{Y G B}=\frac{2}{\left(2+\lambda / \lambda_{O}\right)\left(2+3 \mu / \mu_{O}\right)}
$$

for a spherical droplet for $\mathrm{Ma} \rightarrow 0$. It is known that the actual terminal velocity decreases as Ma increases (Ma and Bothe, 2011; Liu et al., 2012). Figure 6 shows the current results of the terminal velocity and the reported ones (Balasubramaniam and Subramanian, 


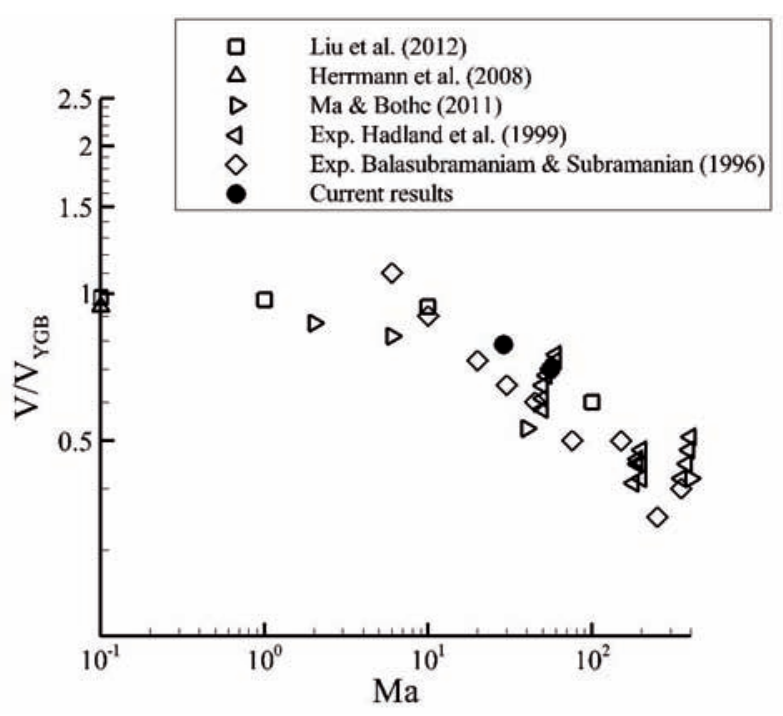

FIG. 6: Terminal velocity of thermocapillary migration.

1996; Hadland et al., 1999; Herrmann et al., 2008; Ma and Bothe, 2011; Liu et al., 2012). The current results are in good agreement with the reported results.

By the two validation cases in this section, it can be concluded that the code can produce physically correct results for phenomena related to convective heating of an emulsion droplet prior to microexplosion/puffing.

\section{RESULTS AND DISCUSSION}

\subsection{Flow Field Structures}

The ambient convective air induces the liquid flow inside a droplet through the shear stress on the droplet surface. The strength of this induced flow determines the temperature distribution inside. Figure 7 shows the temporal history of the positions of three water sub-droplets for Case 3. The normalized time $t^{*}$ is defined as $t^{*}=t U / D$. In this figure, the rotational motion in the velocity field is clearly observed. A water subdroplet in the upstream side (\#1) is pushed toward the downstream along the droplet surface. A sub-droplet at the center (\#2) is initially pushed forward and then to the side. A sub-droplet in the shoulder region (\#3) is initially pushed toward the inside and then forward. All these trajectories indicate that a pair of circular motions exist inside the parent droplet. Note that droplet oscillation and inclination have made the flow field slightly asymmetric. This oscillation is gradually attenuated due to the viscosity of the oil (see the following 3D case). 

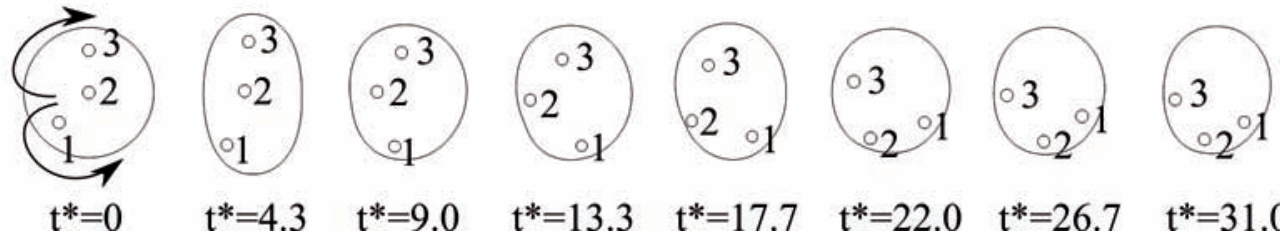

$$
\mathrm{t}^{*}=4.3 \quad \mathrm{t}^{*}=9.0
$$

$\mathrm{t}^{*}=13.3$

$t^{*}=17.7 \quad t^{*}=22.0$

$\mathrm{t}^{*}=26.7 \quad \mathrm{t}^{*}=31.0$

FIG. 7: Time sequence of water sub-droplet motion (Case 3). The air flow is from the left to the right.

A similar velocity field is also developed in the 3D case (Case 1). Figure 8 shows the streamlines at $t^{*}=11.6$ on a plane including the center of gravity of the droplet. The circular streamlines inside the droplet are observed, as well as the recirculation zones behind the droplet. This 3D velocity field inside the droplet is similar to that of Hill's vortex (Sirignano, 2010). As the droplet Reynolds number is initially 30, the secondary vortex formation is not evident in the aft region of the droplet where gas flow separation occurs. For liquid fuel droplets in a combustor, where the liquid density is much larger than the gas density, this secondary vortex effect is minor (Sirignano, 2010).

The vortex core location is slightly inclined toward the windward side. This phenomenon has been also reported by Dwyer and Sanders (1984), Dandy and Leal (1989), and Bergeles et al. (2014). Figure 9(a) shows the vortex core location in terms of the forward angle and the distance from the central axis. Although Re is different among these cases, the vortex core locations are not sensitive to Re. In the current emulsion droplet case, the asymmetry makes water sub-droplets gather relatively toward the windward side.

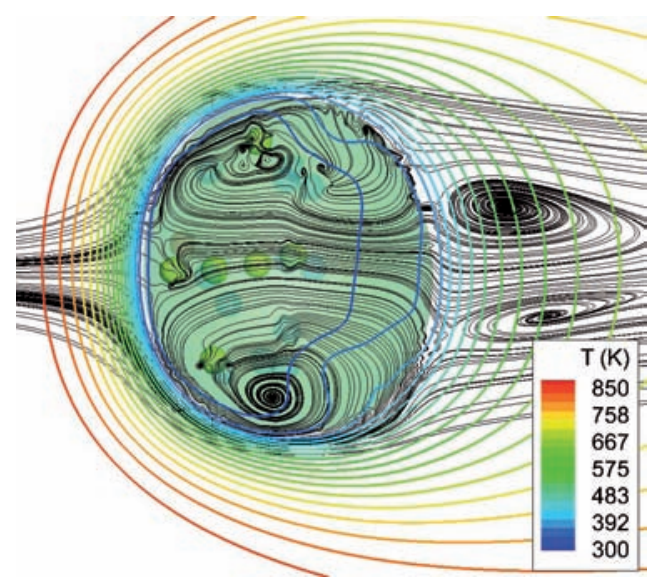

FIG. 8: Streamlines in 2D cut plane at $t^{*}=11.6$. The color contours show temperature field on a plane including the center of gravity of the droplet. 


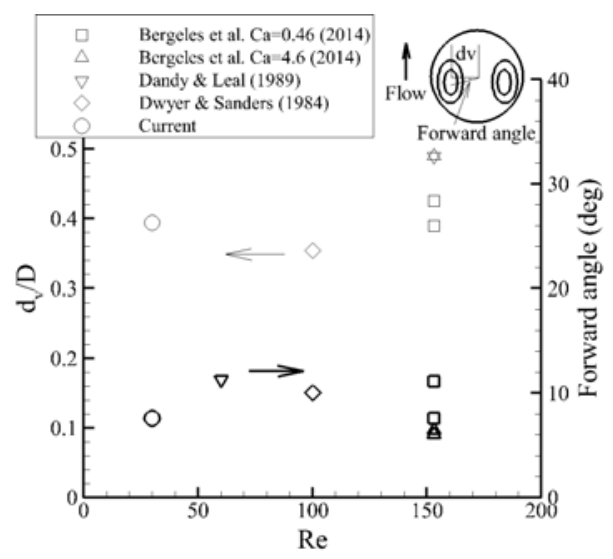

(a)

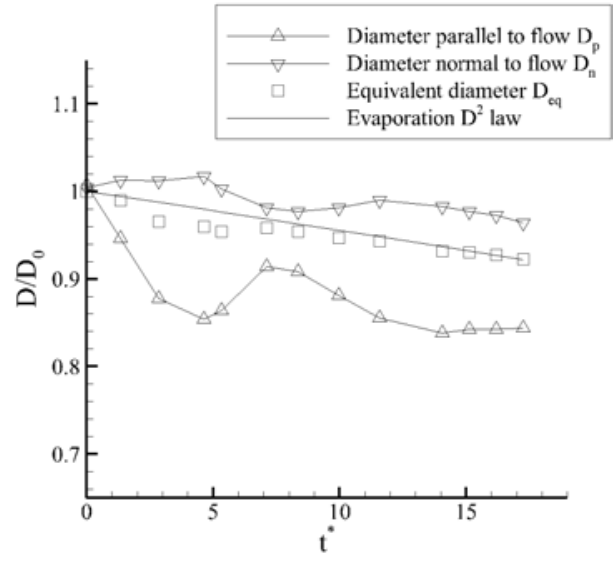

(b)

FIG. 9: Vortex core location and initial shape oscillation; (a) internal vortex core location, (b) droplet shape oscillation.

Droplet shape oscillation is triggered by the initial impact of the gas flow. The theoretical linear oscillation period $T_{o s c}$ is given by (Lamb, 1945)

$$
T_{\text {osc }}=\frac{2 \pi}{\omega}, \quad \omega^{2}=\frac{n(n-1)(n+1)(n+2)}{(n+1) \rho_{L}+n \rho_{G}} \frac{\sigma}{a_{0}^{3}}
$$

where $a_{0}$ is the equilibrium droplet radius and the lowest mode is $n=2$. In the current case, the oscillation period is $T_{o s c}^{*} \sim 7.2$ in the nondimensional form. Figure 9 (b) shows the observed droplet shape oscillation. The droplet deformation in the flow-parallel direction is more extensive than in the flow-normal direction due to the stronger dynamic pressure on the windward surface. The deformation amplitudes are small and the oscillation period is in good agreement with the theoretical value of 7.2 calculated by Eq. (14). The equivalent diameter $D_{e q}=\left(D_{p} D_{n}^{2}\right)^{1 / 3}$ where $D_{p}$ and $D_{n}$ are the diameter parallel and normal to the flow, respectively, gradually decreases due to evaporation. In this figure, a rough estimation of the evaporation by the $D^{2}$ law with the convective effect (Law, 1982; Sirignano, 1983; Lefebvre, 1998; Sirignano, 2010) is also plotted, which is given by

$$
d\left(D^{2}\right) / d t=-\left(1+0.22 \operatorname{Re}^{0.5}\right)\left[\left(\lambda_{G} / 8 \rho_{L} c_{p, G}\right) \ln \left(1+B_{H}\right)\right]
$$

where $B_{H}=c_{p, G}\left(T_{\infty}-T_{s}\right) h_{l}^{-1}$ is the heat transfer number. The observed evaporation follows this prediction. The shape oscillation is soon attenuated by the liquid viscosity, and therefore it does not have a significant impact on the droplet internal circulation and heating. The droplet does not return to a perfect sphere due to the dynamic pressure on the windward surface (We $\sim 0.5$ ), but this does not affect the overall behavior of heating. 


\subsection{Inner-Droplet Temperature Field}

In the above discussion, it has been seen that the internal circulation develops inside the emulsion droplet. The temperature field is examined in this subsection. The relation between the velocity and temperature fields, which is determined by the Peclet number, is interesting and important.

Figure 10 shows the temperature fields of Case 3 (a 2D emulsion droplet), Case 5 (a 2D oil droplet), Case 6 (a 2D water droplet), Case 1 (a 3D emulsion droplet), and Case 2 (a 3D emulsion droplet) at the initial phase. The high temperature initially progresses toward the inside from the aft shoulder region due to the internal circulation of the velocity field. Due to the higher thermal diffusivity of water $\left(\alpha_{W}=14.7 \times 10^{-8} \mathrm{~m}^{2} / \mathrm{s}\right)$ compared to that of the oil $\left(\alpha_{O}=8.21 \times 10^{-8} \mathrm{~m}^{2} / \mathrm{s}\right)$, the temperature distribution in

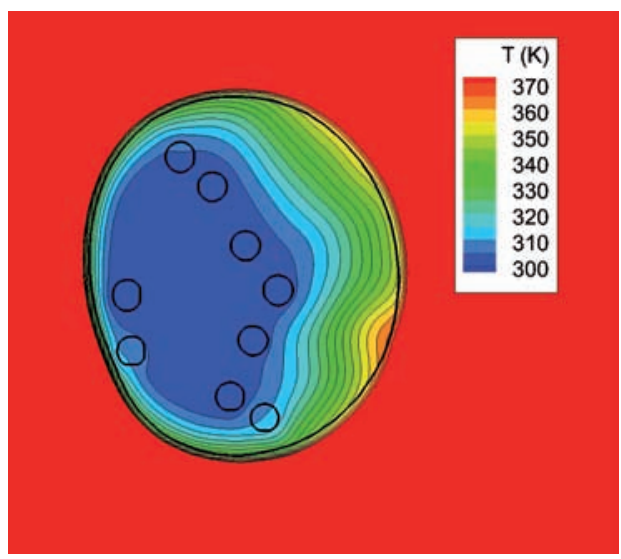

(a)

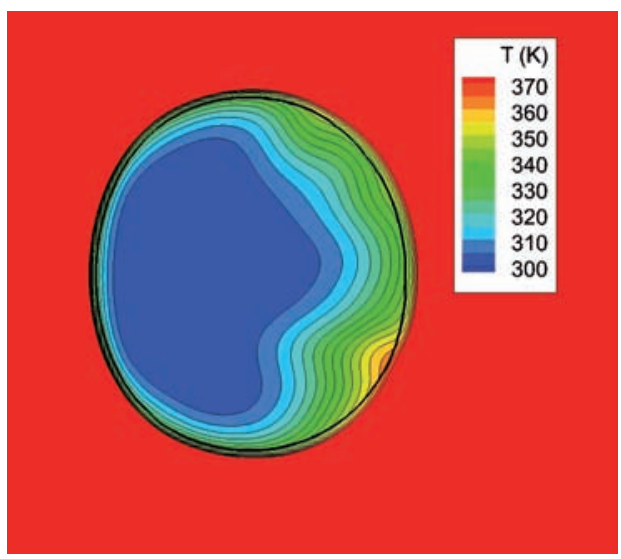

(b)

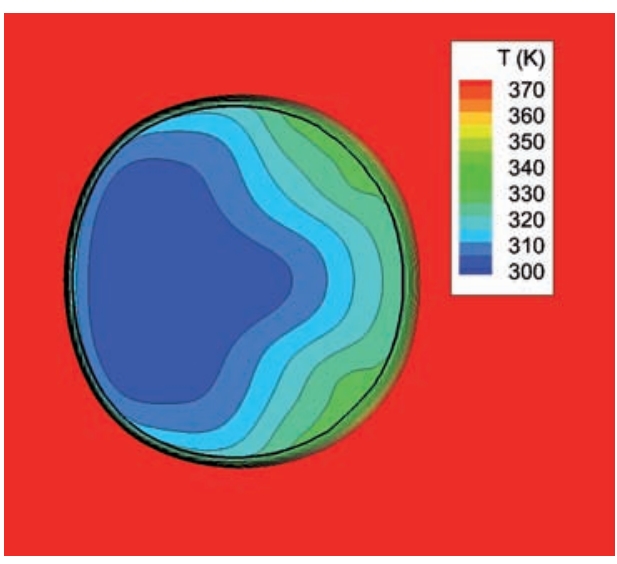

(c)

FIG. 10 


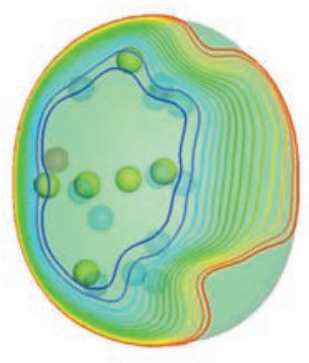

(d)

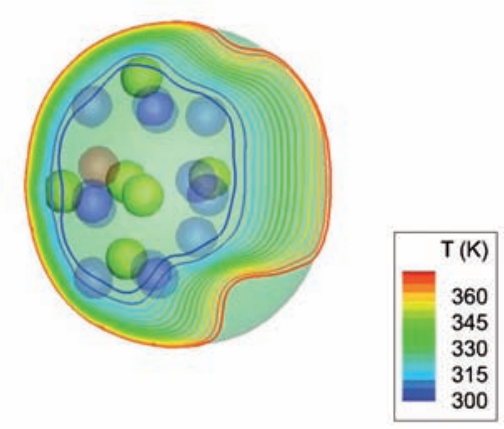

(e)

FIG. 10: Initial transient temperature field evolution. In (d) and (e), the color of water sub-droplet surface is given only to show the multiple level-set functions and they have no distinctions physically. (a) Emulsion (Case 3) at $t^{*}=13$, (b) oil (Case 5) at $t^{*}=13$, (c) water (Case 6) at $t^{*}=13$, (d) emulsion in 3D (Case 1) at $t^{*}=11.6$, (e) emulsion in 3D (Case 2) at $t^{*}=8.5$.

the water droplet case is more diffused in the streamline-normal direction [Fig. 10(c)] than in the oil droplet case [Fig. 10(b)] after the same time span. The temperature field of the emulsion case in Fig. 10(a) is similar to that of the oil case [Fig. 10(b)] since the Stokes numbers of the water sub-droplets are nearly zero and these water sub-droplets follow the local oil motion. Cases 1 and 2 in 3D configurations [Figs. 10(d) and 10(e)] also exhibit the temperature distribution similar to that of Case 3 [Fig. 10(a)].

At a later stage, the temperature distribution becomes more diffused due to the further progressed heat transfer inside the droplet. Figure 11 shows the temperature fields of the emulsion droplet (Case 3) and the oil droplet (Case 5). The lowest temperature remains in the windward region, and the locations correspond to the vortex cores, but the shape of the temperature distribution is not perfectly similar to the shape of Hill's vortex. The internal circulation size/orientation becomes somewhat tilted due to flow disturbances coupled with droplet shape deformation (Sirignano, 2010). Once the asymmetry occurs, it will not be easy to recover the perfect symmetry again due to internal circulation.

To further understand the flow regime, the droplet Reynolds number Re and the liquid Peclet number $\mathrm{Pe}_{L}$ are shown in Fig. 12. The droplet Reynolds number Re gradually decreases due to the air drag force on the droplet, as shown in Fig. 12(a). The relatively faster deceleration in the $3 \mathrm{D}$ case is simply explained by the equation of motion. Newton's second law gives $\left(\rho_{L} \pi a^{2} L\right) \cdot \ddot{x}_{2 D}=-0.5 \rho_{G} U_{G}^{2}(a L) c_{D}$ in $2 \mathrm{D}$ configurations 

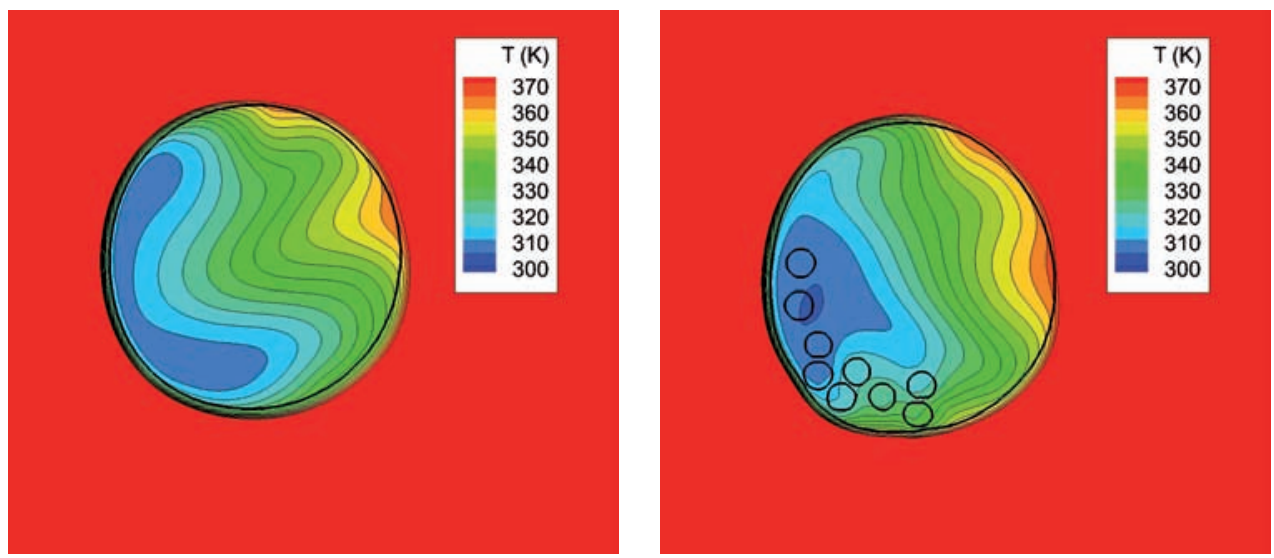

FIG. 11: Temperature distributions for oil droplet (Case 5) at $t^{*}=39$ (left) and for emulsion droplet (Case 3) at $t^{*}=41.7$ (right). The air flow is from the left to the right.

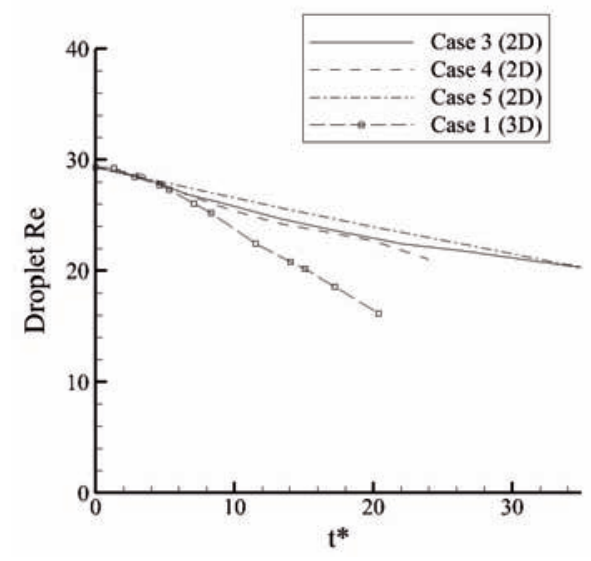

(a)

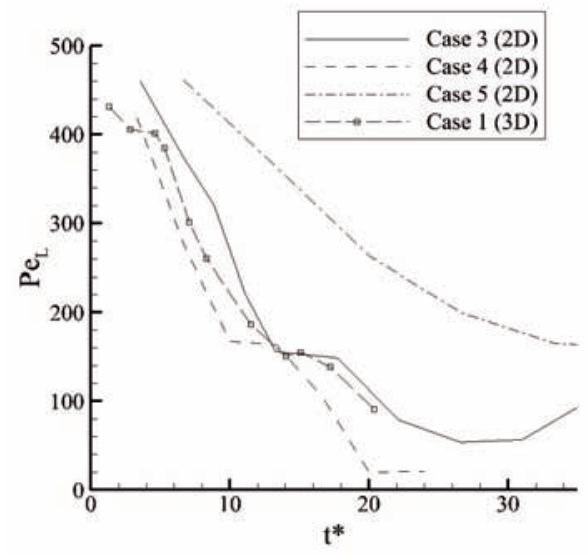

(b)

FIG. 12: Time history of the droplet Reynolds and liquid Peclet numbers.

and $\left(4 \rho_{L} \pi a^{3} / 3\right) \cdot \ddot{x}_{3 D}=-0.5 \rho_{G} U_{G}^{2}\left(\pi a^{2}\right) c_{D}$ in $3 \mathrm{D}$ configurations. The deceleration is faster in the 3D case since $\left|\ddot{x}_{3 D} / \ddot{x}_{2 D}\right|=3 \pi / 4>1$, which is based on the fact that the drag coefficients are almost the same, $c_{D} \sim 3.0$ around $\operatorname{Re} \sim 30$ for both a sphere and a cylinder (Munson et al., 1994).

The liquid Peclet number $\mathrm{Pe}_{L}$ varies more drastically. Figure 12(b) shows the observed $\mathrm{Pe}_{L}$ for the 2D emulsion (Cases 3 and 4) and the 2D oil (Case 5) droplets and the $3 \mathrm{D}$ emulsion droplet (Case 1). The liquid surface velocity $U_{s}$ is used for this $\mathrm{Pe}_{L}$ calculation. In Case 1 , for example, $U_{s}=0.832 \mathrm{~m} / \mathrm{s}$ when $\operatorname{Re}=28.5$. This value is close to 
the estimation given in Abramzon and Sirignano (1989) as $U_{s}=a U\left(\mu_{G} / \mu_{L}\right) \cdot \operatorname{Re} \cdot C_{F}$, where $a=1 / 32$ and $C_{F}=12.69 \mathrm{Re}^{-2 / 3}$. This gives $U_{s}=1.04 \mathrm{~m} / \mathrm{s}$. For all the cases, the peak $\mathrm{Pe}_{L}$ is around 400-500. Then $\mathrm{Pe}_{L}$ decreases due to the decrease of the shear force caused by the development of a boundary layer around the droplet. Abramzon and Sirignano (1989) and Sirignano (2010) argue that a complete Hill's vortex shape of $T$ is typically observed for $\mathrm{Pe}_{L} \sim \mathrm{O}(1000)$. For the cases in the present study, $\mathrm{Pe}_{L}$ decreases to 150-400 within a short period before $t^{*}<10$. This means that the formation of a complete Hill's vortex in the temperature field is unlikely under the current conditions.

In the case of the oil droplet (Case 5), the decrease of $\mathrm{Pe}_{L}$ is the slowest. Since the emulsion droplets have embedded water sub-droplets and the density of water is larger than that of the oil, the internal circulation motion is relatively more difficult to develop for emulsion droplets. This can also be confirmed by comparing Cases 3 and 4. Case 4 has larger water sub-droplets and the decrease of $\mathrm{Pe}_{L}$ is faster. Therefore, for emulsion droplets, the temperature field is relatively less affected by the convective effect, compared to mono-component droplets.

\subsection{Marangoni Effect on a Parent Oil Droplet}

Generally, the surface tension coefficient becomes smaller as the temperature rises. During the convective droplet heating, the surface temperature of a parent droplet varies spatially, especially at the initial time. Therefore, the Marangoni effect may have some effect initially. It should be stressed that the discussion here is about the Marangoni effect on the parent droplet surface, not on the thermocapillary migration of embedded water sub-droplets inside an emulsion droplet. The latter effect is minor for the cases considered in the present study (see Section 1).

To consider the variation of $\sigma$ with temperature, $\sigma$ of the oil is given by $\sigma_{O}=$ $[27.12-0.088(T-273)] \times 10^{-3} \mathrm{~N} / \mathrm{m}$ (Jasper, 1974). Figure 13 shows the comparison of the temperature distribution inside the oil and emulsion droplets with/without the Marangoni effect. The internal circulation is slightly enhanced with the Marangoni effect in both cases, similarly in Shih and Megaridis (1996). Figure 13(c) shows the surface temperature in the early transient period. The steep change in the temperature in the leeward region $\left(120^{\circ}-135^{\circ}\right)$ causes the imbalance in the surface tension force, and the overall surface tension imbalance works in the direction to enhance the internal circulation. However, this effect is not strong enough to form Hill's vortex in the temperature field for the current $\mathrm{Pe}_{L}$ range, and as the time passes this effect is attenuated. Thus, this effect is considered to be secondary here.

\subsection{Water Evaporation from the Parent Droplet Surface}

In Case 4 where the water sub-droplet size is large, it has been observed that a water subdroplet reaches the parent droplet surface and evaporates there, as indicated by the arrow 

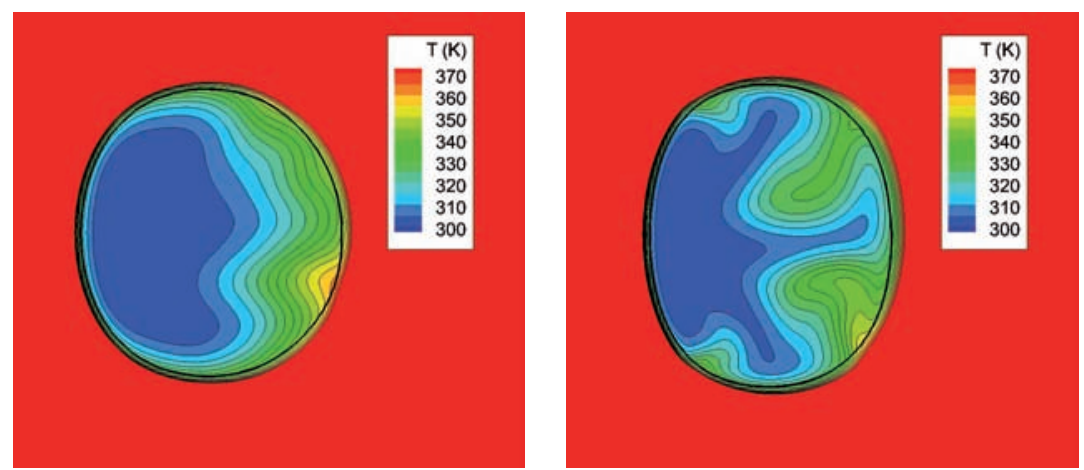

(a)
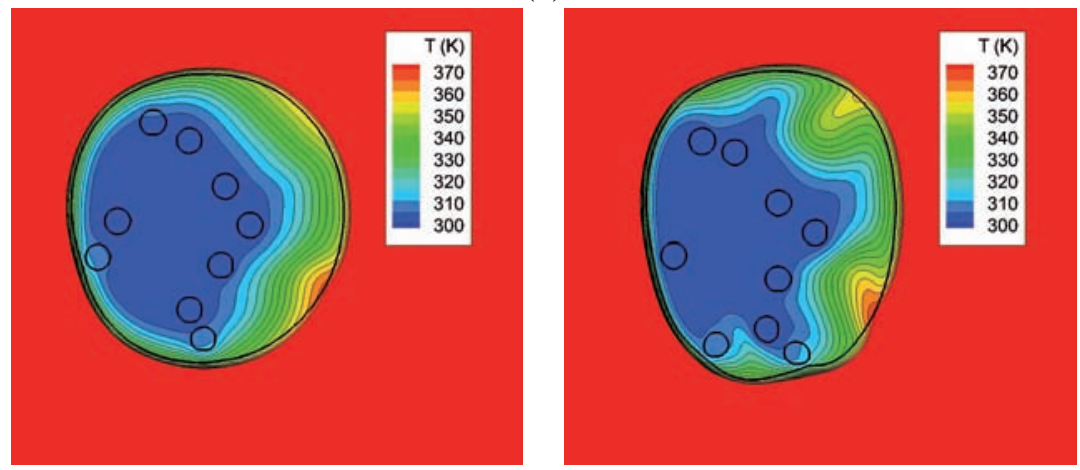

(b)

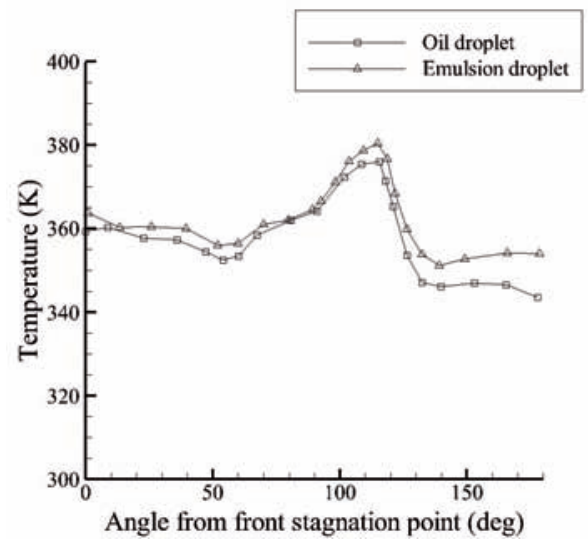

(c)

FIG. 13: Difference in circulation acceleration due to the Marangoni effect. (a) Oil droplet at $t^{*}=17.3$. Left: Case 5 and right: Case 5M. (b) Emulsion droplet at $t^{*}=$ 10.6. Left: Case 3 and right: Case 3M. (c) Surface temperature distribution at $t^{*}=4.4$ for Cases 3 and 5. 
in Fig. 14. It has been argued that water evaporation (or boiling) at the parent oil droplet would affect the droplet heating characteristics due to the heat absorption by the latent heat of evaporation of water (Law et al., 1980; Law, 1982; Sirignano, 2010). Especially in the distillation limit regime (Law, 1977, 1982; Law et al., 1980), where fresh water is continuously supplied from the inside due to the very strong internal circulation, it is considered that this effect is non-negligible. In the current study, however, the internal circulation is not strong enough to supply water sub-droplets to the surface continuously. For Cases 1 and 3 where the size of water sub-droplets is smaller, water evaporation at the oil parent droplet surface is not commonly observed.

Even for Case 4 in Fig. 14, the evaporation is local and its influence is confined to the vicinity of this evaporating water sub-droplet. The oil droplet surface temperature away from the evaporating water sub-droplet can still rise, and the heat from the gas can be transferred into the oil droplet. It is expected that the water sub-droplet evaporating at the oil droplet surface will be finally depleted. Afterwards, the emulsion droplet surface can be viewed again as a no-water layer with moderate internal circulation (Sheng et al., 1994). Therefore, in the modeling study in the next subsection, water evaporation at the parent oil droplet surface is not considered as a primary phenomenon to be modeled, as in Tarlet et al. (2009) and Watanabe et al. (2010).

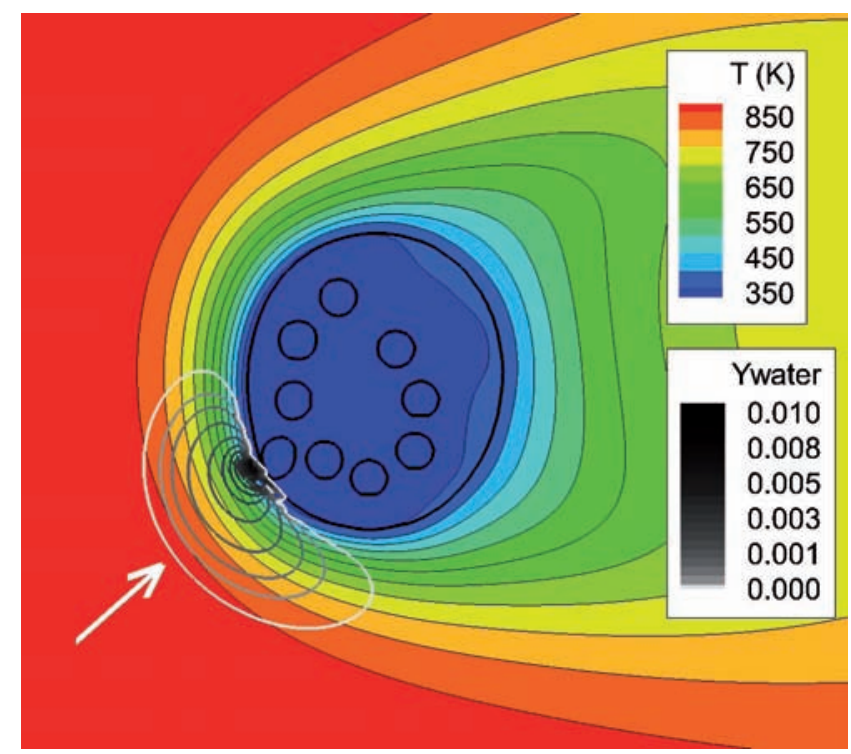

FIG. 14: Evaporating water sub-droplet on the surface, indicated by the arrow. The background color contours show the temperature and the superposed contours represent the mass fraction of water vapor. Note that the magnitude of water mass fraction contour range is small. 


\subsection{Modeling Temperature Distribution inside an Emulsion Droplet under Convective Heating}

From the results in Section 4.2, the liquid Peclet number $\mathrm{Pe}_{L}$ plays an important role in inner-droplet temperature distribution for emulsion droplets as well as for monocomponent droplets. Figure 15 illustrates the temperature field at various Peclet numbers. The current results are in the regime of $100<\mathrm{Pe}_{L}<1000$.

In the regime of $\mathrm{Pe}_{L}>1000$, the vortex model proposed by Sirignano and coworkers (Prakash and Sirignano, 1978, 1980; Tong and Sirignano, 1982; Sirignano, 1983, 2010; Abramzon and Sirignano, 1989) gives a good approximation. Both the velocity and temperature fields become similar to Hill's vortex since the heat transfer along the streamline direction is faster than the heat conduction normal to the streamlines. The temperature equation can be formulated in the conduction form as (Prakash and Sirignano, 1978, 1980)

$$
\frac{\partial \bar{T}}{\partial \tau}=a(\varphi, \tau) \frac{\partial^{2} \bar{T}}{\partial \varphi^{2}}+b(\varphi, \tau) \frac{\partial \bar{T}}{\partial \varphi},
$$

where $\tau=\alpha t / a_{0}^{2}, \bar{T}=\left(T_{a v}-T_{0}\right) /\left(T_{b}-T_{0}\right), \varphi$ is the coordinate perpendicular to the streamlines, $T_{a v}$ the average temperature in the streamline direction, and $T_{b}$ the boiling temperature. It can be simplified to

$$
\frac{\partial \bar{T}}{\partial \hat{\tau}}=\varphi \frac{\partial^{2} \bar{T}}{\partial \varphi^{2}}+[1+C(\hat{\tau}) \varphi] \frac{\partial \bar{T}}{\partial \varphi},
$$

where $d \hat{\tau}=\left(b_{1} / b_{0}\right)\left(a_{0} / a\right)^{2} d \tau$ and $C(\hat{\tau})=2\left(a_{0} / a\right)^{3 / 2} \cdot d\left(a_{0} / a\right)^{3 / 2} / d \hat{\tau}$ with $b_{0} \sim 0.3$ and $b_{1} \sim 5.4$ (Tong and Sirignano, 1982).

Meanwhile, in the regime of $\mathrm{Pe}_{L}<100$, the internal circulation is weak, although it slightly enhances the inner-droplet heat transfer. This augmented heat transfer is assumed to be symmetric, namely, it has no directional orientation. Abramzon and Sirignano (1989) have proposed the effective conductivity (EC) model for such a case. The modulation due to the weak convective effect is modeled by the augmented effective thermal conductivity, which is given as (Abramzon and Sirignano, 1989)

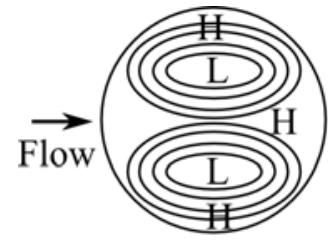

$\mathrm{Pe}_{\mathrm{L}}>1000$

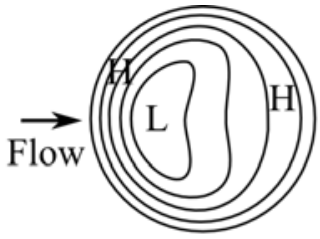

$100<\mathrm{Pe}_{\mathrm{L}}<1000$

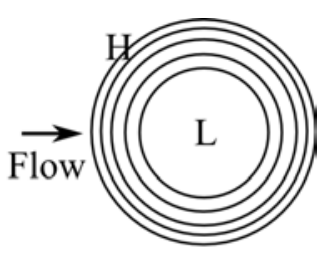

$\mathrm{Pe}_{\mathrm{L}}<100$

FIG. 15: Typical temperature field in a droplet at various liquid Peclet numbers $\mathrm{Pe}_{L} \cdot \mathrm{H}$ denotes high temperature and $L$ low temperature. 


$$
\begin{aligned}
& \lambda_{e f f}=\lambda \cdot \chi \\
& \chi=1.86+0.86 \tanh \left[2.245 \log _{10}\left(\mathrm{Pe}_{L} / 30\right)\right] .
\end{aligned}
$$

For example, for $\mathrm{Pe}_{L}=10, \chi=1.18$ and for $\mathrm{Pe}_{L}=100, \chi=2.57$. A similar idea can be found in Tarlet et al. (2009).

Under the current flow conditions for typical spray droplets in a combustor, the results in Sections 4.1. and 4.2. suggest that $\mathrm{Pe}_{L}$ lies between the above two limits $\left(100<\mathrm{Pe}_{L}<500\right)$. The internal circulation is not strong enough to make the temperature field similar to Hill's vortex, but the temperature distribution is inclined toward the windward direction due to the internal circulation (see Fig. 15). To take the above observation into consideration of the modeling, two effects are added here. The first is to modify the effective thermal conductivity to have an angular orientation. Defining an angle $\theta(0<\theta<\pi)$ which is measured from the rear stagnation point,

$$
\lambda_{e f f, m}=\lambda_{e f f} \cdot[2.74 \exp (-1.076 \theta)]
$$

is an optimized effective thermal conductivity for Case 1 . The second effect to be added is the eccentricity in the temperature field, as schematically shown in Fig. 16. We call this method the effective conductivity with modified eccentricity (ECME) model.

The temperature equation to be solved in the nondimensional form is (Chiu and Chen, 1996)

$$
\partial \bar{T} / \partial \tau=\nabla^{2} \bar{T}
$$

where $\tau=\alpha t / r_{0}^{2}$ and $\bar{T}=\left(T-T_{o}\right) /\left(T_{i}-T_{o}\right)$. The subscript $o$ denotes the outer surface of the parent droplet. The inner sphere $\left(r_{i}\right)$ is hypothetical and represents the vortex core. $\varepsilon$ is the normalized eccentricity $(0<\varepsilon<1)$ for the core location. The Laplacian operator on the right-hand side is

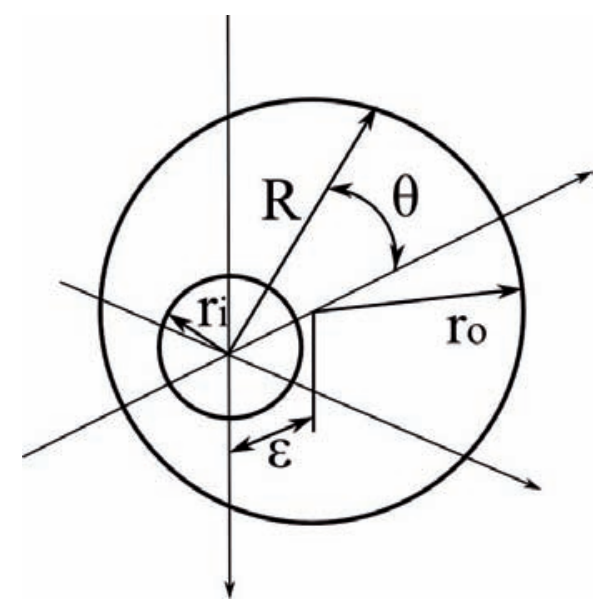

FIG. 16: Coordinates for the eccentric spherical system. 


$$
\begin{aligned}
\nabla^{2} & =\left[\left(\frac{\partial \eta}{\partial r}\right)^{2}+\frac{1}{\pi^{2} r^{2}}\left(\frac{\partial \eta}{\partial \theta}\right)^{2}\right] \frac{\partial^{2}}{\partial \eta^{2}}+\left[\frac{2}{\pi^{2} r^{2}}\left(\frac{\partial \eta}{\partial \theta}\right)\right] \frac{\partial^{2}}{\partial \eta \partial \theta}+\left[\frac{1}{\pi^{2} r^{2}}\right] \frac{\partial^{2}}{\partial \theta^{2}} \\
& +\left[\frac{2}{r}\left(\frac{\partial \eta}{\partial r}\right)+\frac{1}{\pi^{2} r^{2}}\left(\frac{\partial^{2} \eta}{\partial \theta^{2}}\right)+\frac{\cot (\pi \theta)}{\pi r^{2}}\left(\frac{\partial \eta}{\partial \theta}\right)\right] \frac{\partial}{\partial \eta}+\left[\frac{\cot (\pi \theta)}{\pi r^{2}}\right] \frac{\partial}{\partial \theta}
\end{aligned}
$$

where

$$
\eta=\frac{r-r_{i}}{R(\theta)-r_{i}}, \quad R(\theta)=\sqrt{r_{o}^{2}-\varepsilon^{2} \sin ^{2} \theta}+\varepsilon \cos \theta,
$$

as shown in Fig. 16. To minimize the effect of the hypothetical inner core, the radius of the inner core is made small $\left(r_{i}=0.05 r_{o}\right.$ in Fig. 17). The eccentricity is determined by the simulation results in Sections 4.1 and 4.2. It is not sensitive to $\mathrm{Pe}_{L}$, and set as $\varepsilon=0.45$ here. The extension to emulsion droplets can be included by introducing an averaged thermal diffusivity $\bar{\alpha} \cdot \bar{\alpha}$ is determined by the mass fraction of the oil and water, and becomes slightly larger than the thermal diffusivity of the oil. $\bar{\lambda}=\sum Y_{i} \lambda_{i}, \bar{c}_{p}=$ $\sum Y_{i} c_{p, i}$, and $\bar{\rho}^{-1}=\sum Y_{i} \rho_{i}^{-1}$ are used for averaging.

Figure 17 shows the model predictions with $80 \times 80$ grid points for Case 1 . The boundary condition at the droplet surface is given by a prescribed surface temperature $(380 \mathrm{~K})$ for simplicity. A zero-gradient condition is imposed at the inner core. The EC model in Fig. 17(b) gives an acceptable approximation. The temperature range is in good agreement with the simulation result [Fig. 17(a)]. However, the inclination of the temperature field induced by the internal circulation cannot be reproduced. The ECME model gives a better result, as shown in Fig. 17(c). The temperature field becomes closer to that shown in Fig. 17(a). At a later time $\left(t^{*}=20.4\right)$, the ECME model also predicts a similar temperature field where the heat diffusion progresses further from the rear side [Fig. 17(e)]. Note that the slightly wrinkled temperature contour lines in Fig. 17(d) are due to the existence of the water sub-droplets, for which detailed modeling is not intended here due to the consideration of the model complexity. It can be seen that the temperature prediction becomes possible in an emulsion spray under combustor conditions with the ECME model. The temperature prediction determines the bubble nucleation, which is one important process for hybrid high-fidelity multiscale simulation of emulsion spray processes with microexplosion/puffing modeled.

The ECME model is 2D (in the radial and angular directions) and therefore the computational cost is higher. Note that the "2D" here means that the axisymmetric ECME model has two independent variables, $R$ and $\theta$, to model the temperature distribution inside a $3 \mathrm{D}$ emulsion droplet under convective heating. The cost consideration is important, because the inner-droplet temperature distribution model developed in the present study is intended to be incorporated into an Eulerian-Lagrangian code MultiPLESTaR (Xia and Luo, 2009, 2010; Xia et al., 2013) to perform high-fidelity multiscale simulation of emulsion fuel spray processes under microexplosion/puffing conditions. In MultiPLESTaR, droplets are approximated by point particles and only one data of the double-precision kind is needed to store droplet temperature under the assumption of an 


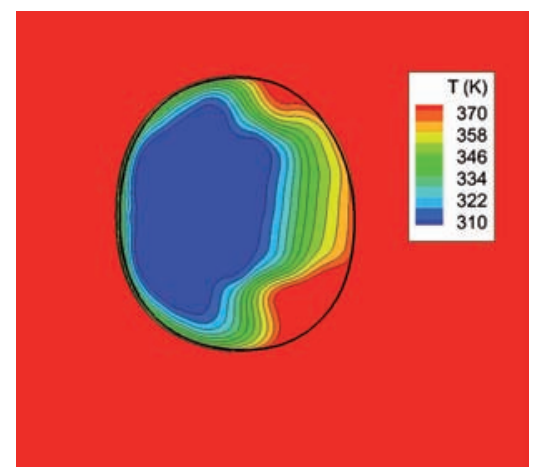

(a)

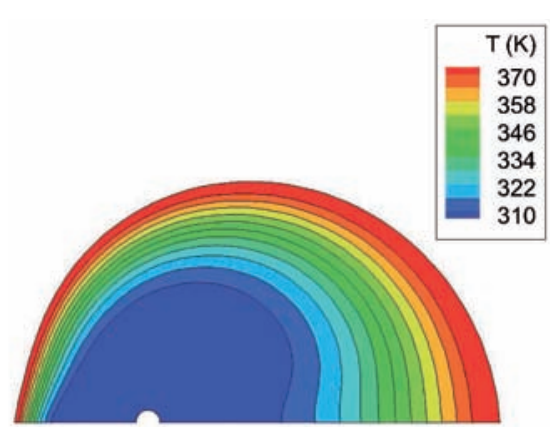

(c)

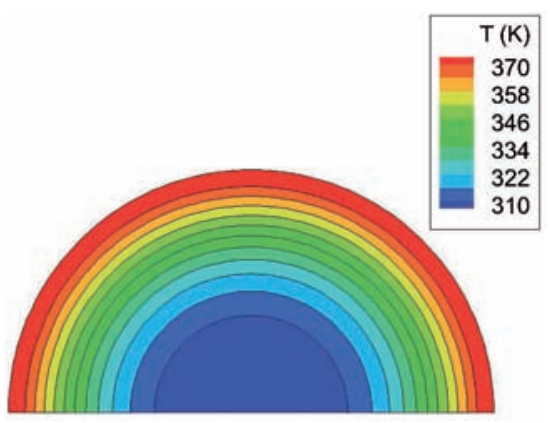

(b)

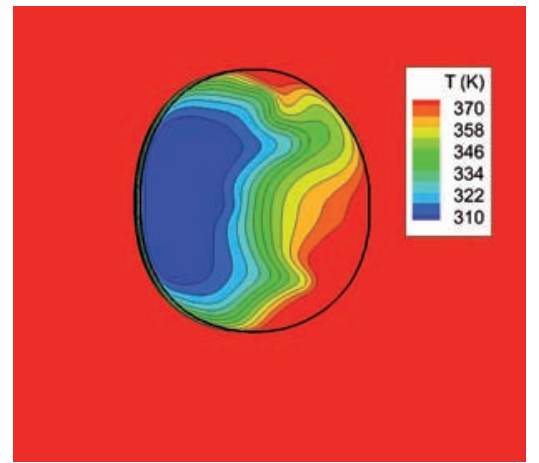

(d)

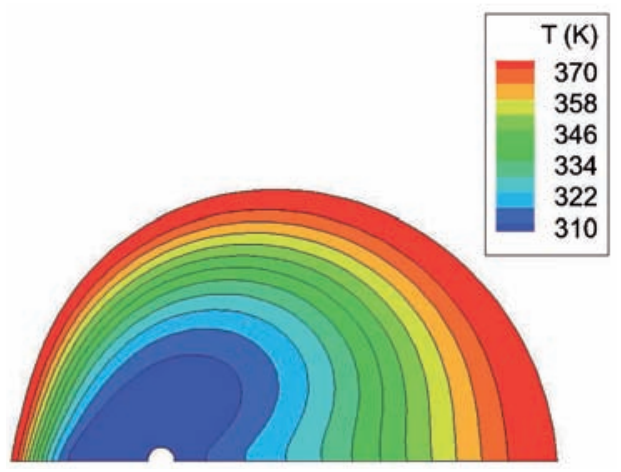

(e)

FIG. 17: Temperature distribution by models. (a) Simulation result [3D Case 1, Fig. 11(d)] at $t^{*}=11.6$, (b) ECM model at $t^{*}=11.6$, (c) ECME model at $t^{*}=11.6$, (d) simulation result (3D Case 1) at $t^{*}=20.4$, and (e) ECME at $t^{*}=20.4$. In (c) and (e), the small white inner circle shows the position of the inner core center. (a) $t^{*}=11.6$, (b) EC at $t^{*}=11.6$, (c) ECME at $t^{*}=11.6$, (d) $t^{*}=20.4$, (e) ECME at $t^{*}=20.4$. 
infinite thermal conductivity. Therefore, the 2D ECME model will increase the size of the data structure, and therefore increase the requirement of computer memory size and the message size of droplets transferred between CPU cores.

Figure 18 compares the effect of grid resolution in the ECME model, where $i \times j$ means $i$ points in the radial direction and $j$ points in the angular direction. The $80 \times 80$ case is the baseline reference case as shown in Fig. 17. The root mean square (rms) error is calculated as the magnitude of deviation from the result of the $80 \times 80$ case, namely,

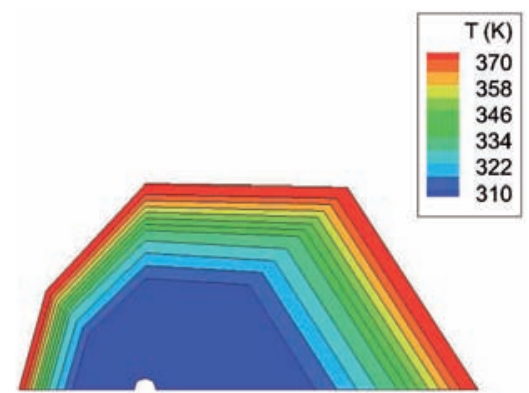

(a)

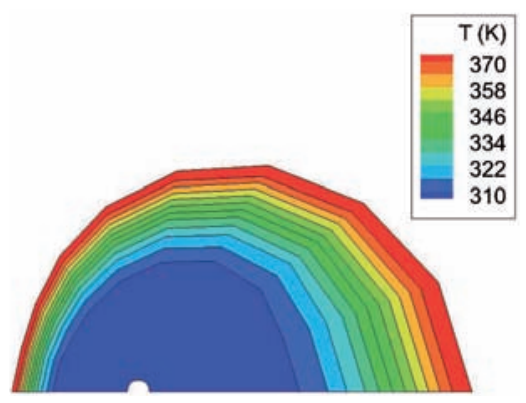

(b)

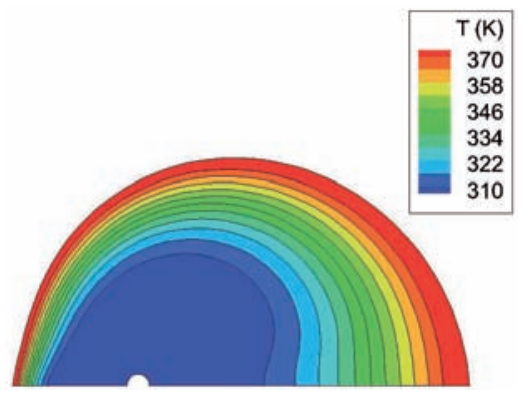

(c)

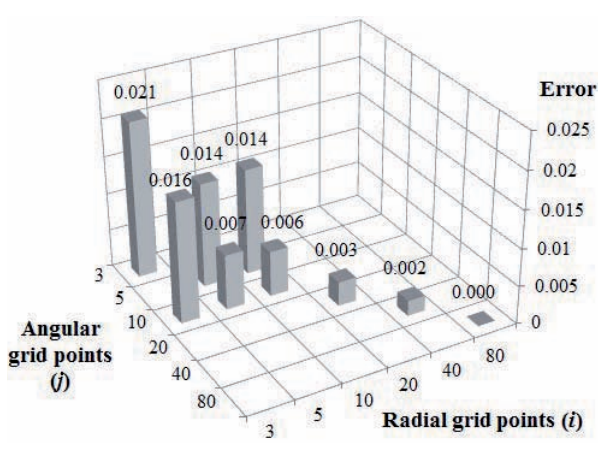

(d)

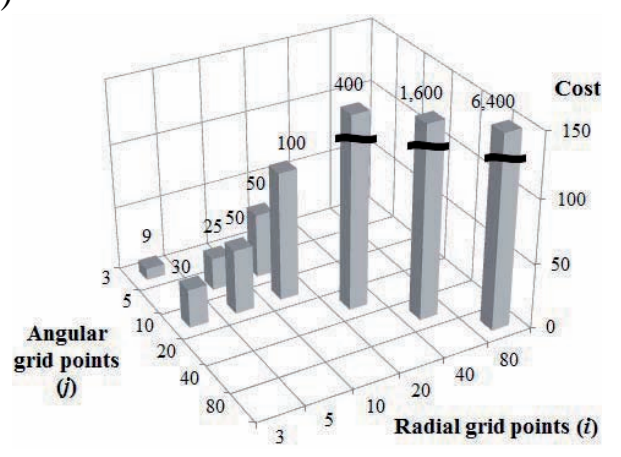

(e)

FIG. 18: Temperature distribution and error/cost analysis for the ECME model at $t^{*}=$ 11.6 with different grid systems. (a) $5 \times 5$, (b) $10 \times 10$, (c) $80 \times 80$, (d) error, (e) cost. 


$$
\text { error }=\sqrt{\frac{1}{n} \sum\left(1-\frac{T}{T_{80}}\right)^{2}},
$$

where $n$ is the total number of grid points and $T_{80}$ is the temperature data of the $80 \times$ 80 case. The cost is assumed to be proportional to the total grid number. Even for the coarse cases of $5 \times 5$ and $10 \times 10$, the distinct feature of the temperature distribution can be seen [Figs. 18(a) and 18(b)], for which the error is $1.4 \%$ and $0.6 \%$, respectively. The cost is higher than in the point-temperature model or in the 1D EC model, but the difference may be fairly marginal. The overall trend shows that increasing the number of the angular grid points improves the accuracy better than increasing the number in the radial direction.

\section{CONCLUDING REMARKS}

To accurately model microexplosion of emulsion fuel droplets, a model has been proposed to predict the temperature distribution inside an emulsion droplet under convective heating. The inner-droplet temperature field is vitally important since it largely determines the initiation of microexplosion/puffing, i.e., the onset of nucleation in superheated water sub-droplets. To assist the model development, convective heating of a water-in-oil emulsion droplet has been investigated by detailed numerical simulation. Direct simulation of emulsion droplet heating has been made possible by the MLE method, in which multiple level-set functions are utilized to avoid coalescence of embedded water sub-droplets in an emulsion droplet. It was found that the shear force from the hot ambient gas induces internal circulation and heat transfer is affected by this motion. For a substantial time period, the transient heating effect remains and the temperature field becomes stratified. Under the flow regime considered in the present study, i.e., $100<$ $\mathrm{Pe}_{L}<500$, the temperature field is affected by internal circulation, but does not follow inner-droplet streamlines. Therefore, both Hill's vortex and the effective conductivity model cannot properly predict the inner-droplet temperature field. A novel model is proposed, which considers the angular dependence of the effective conductivity and the eccentricity of the temperature field. The model results are in good agreement with the simulation results. With the proposed model, the prediction of likely locations of vapor bubble nucleation can be made under the transitional regime of $\mathrm{Pe}_{L}$. This model can be also incorporated into an Eulerian-Lagrangian spray code to enable emulsion fuel spray simulations under microexplosion/puffing conditions.

\section{ACKNOWLEDGMENTS}

Financial support from the Engineering and Physical Sciences Research Council (EPSRC) of the United Kingdom under Grant no. EP/J018023/1 is gratefully acknowledged. This work used the ARCHER UK National Supercomputing Service (http://www.archer.ac.uk).

Volume 26, Issue 6, 2016 


\section{REFERENCES}

Abramzon, B. and Sirignano, W. A., Droplet vaporization model for spray combustion calculations, Int. J. Heat Mass Transfer, vol. 32, pp. 1605-1618, 1989.

Avedisian, C. T. and Glassman, I., Superheating and boiling of water in hydrocarbons at high pressures, Int. J. Heat Mass Transfer, vol. 24, pp. 695-706, 1981.

Avedisian, C. T., The homogeneous nucleation limits of liquids, J. Phys. Chem. Ref. Data, vol. 14, pp. 695-729, 1985.

Balasubramaniam, R. and Subramanian, R. S., Thermocapillary bubble migration-thermal boundary layers for large Marangoni numbers, Int. J. Multiphase Flow, vol. 22, pp. 593-612, 1996.

Bergeles, K., Hardalupas, Y., and Taylor, A. M. K. P., The flow inside and around a falling droplet under various Capillary numbers, In Proc. of ILASS-Europe 2014, 2014.

Brackbill, J. U., Kothe, D. B., and Zemach, C., A continuum method for modeling surface tension, J. Comput. Phys., vol. 100, pp. 335-354, 1992.

Chiang, C. H., Raju, M. S., and Sirignano, W. A., Numerical analysis of convecting, vaporizing fuel droplet with variable properties, Int. J. Heat Mass Transfer, vol. 35, pp. 1307-1324, 1992.

Chiu, C. P. and Chen, W. R., Transient natural convection heat transfer between concentric and vertically eccentric spheres, Int. J. Heat Mass Transfer, vol. 39, pp. 1439-1452, 1996.

Dandy, D. S. and Leal, L. G., Buoyancy-driven motion of a deformable drop through a quiescent liquid at intermediate Reynolds number, J. Fluid Mech., vol. 208, pp. 161-192, 1989.

Dryer, F. L., Water addition to practical combustion systems-concepts and applications, Proc. Combust. Inst., vol. 16, pp. 279-295, 1977.

Dwyer, H. A. and Sanders, B. R., Detailed computation of unsteady droplet dynamics, Proc. Combust. Inst., vol. 20, pp. 1743-1749, 1984.

Fuchihata, M., Ida, T., and Mizutani, Y., Observation of microexplosions in spray flames of light oil-water emulsions (2nd report, influence of temporal and spatial resolution in high speed videography), J. Jpn. Soc. Mech. Eng. (B), vol. 69, pp. 1503-1508, 2003.

Hadland, P. H., Balasubramaniam, R., Wozniak, G., and Subramanian, R. S., Thermocapillary migration of bubbles and drops at moderate to large Marangoni number and moderate Reynolds number in reduced gravity, Exp. Fluids, vol. 26, pp. 240-248, 1999.

Haywood, R. J., Nafziger, R., and Renksizbulut, M., A detailed examination of gas and liquid phase transient processes in convective droplet evaporation, J. Heat Transfer, vol. 111, pp. 495-502, 1989.

Herrmann, M., Lopez, J. M., Brandy, P., and Raessi, M., Thermocapillary motion of deformable drops and bubbles, In Proc. of Summer Program, Center for Turbulence Research, Stanford University, pp. 155-170, 2008.

Himeno, T. and Watanabe, T., Thermo-fluid management under low-gravity conditions (2nd report: free-surface flows driven by surface forces), J. Jpn. Soc. Mech. Eng. (B), vol. 69, pp. 2400-2407, 2003.

Igra, D. and Takayama, K., Numerical simulation of shock wave interaction with a water column, Shock Waves, vol. 11, pp. 219-228, 2001. 
Jasper, J. J., The surface tension of pure liquid compounds, J. Phys. Chem. Ref. Data, vol. 1, pp. 841-1009, 1974.

Kadota, T. and Yamasaki, H., Recent advances in the combustion of water fuel emulsion, Prog. Energy Combust. Sci., vol. 28, pp. 385-404, 2002.

Kunugi, T., Direct numerical algorithm for multiphase flow with free surfaces and interfaces, $J$. Jpn. Soc. Mech. Eng. (B), vol. 63, pp. 1576-1584, 1997.

Lamb, H., Hydrodynamics, New York: Dover, 1945.

Lasheras, J. C., Yap, L. T., and Dryer, F. L., Effect of the ambient pressure on the explosive burning of emulsified and multicomponent fuel droplets, Proc. Combust. Inst., vol. 20, pp. 1761-1772, 1984.

Law, C. K., A model for the combustion of oil/water emulsion droplets, Combust. Sci. Technol., vol. 17, pp. 29-38, 1977.

Law, C. K., Recent advances in droplet vaporization and combustion, Prog. Energy Combust. Sci., vol. 8, pp. 171-201, 1982.

Law, C. K., Lee, C. H., and Srinivasan, N., Combustion characteristics of water-in-oil emulsion droplets, Combust. Flame, vol. 37, pp. 125-143, 1980.

Lefebvre, A. H., Gas Turbine Combustion, 2nd ed., Boca Raton, FL: Taylor and Francis, 1998.

Liu, H., Zhang, Y., and Valocchi, A. J., Modeling and simulation of thermocapillary flows using lattice Boltzmann method, J. Comput. Phys., vol. 231, pp. 4433-4453, 2012.

Ma, C. and Bothe, D., Direct numerical simulation of thermocapillary flow based on the volume of fluid method, Int. J. Multiphase Flow, vol. 37, pp. 1045-1058, 2011.

Munson, B. R., Young, D. F., and Okiishi, T. H., Fundamentals of Fluid Mechanics, New York: Wiley, 1994.

Nas, S. and Tryggvason, G., Thermocapillary interaction of two bubbles or drops, Int. J. Multiphase Flow, vol. 29, pp. 1117-1135, 2003.

NIST (National Institute of Standards and Technology) web database 2011, retrieved April 2014 from http://webbook.nist.gov/chemistry/fluid/.

Prakash, S. and Sirignano, W. A., Liquid fuel droplet heating with internal circulation, Int. J. Heat Mass Transfer, vol. 21, pp. 885-895, 1978.

Prakash, S. and Sirignano, W. A., Theory of convective droplet vaporization with unsteady heat transfer in the circulating liquid phase, Int. J. Heat Mass Transfer, vol. 23, pp. 253-268, 1980.

Segawa, D., Yamasaki, H., Kadota, T., Tanaka, H., Enomoto, H., and Tsue, M., Watercoalescence in an oil-in-water emulsion droplet burning under microgravity, Proc. Combust. Inst., vol. 28, pp. 985-990, 2000.

Sheng, H. Z., Chen, L., Zhang, Z. P., Wu, C. K., An, C., and Cheng, C. Q., The droplet group microexplosions in water-in-oil emulsion sprays and their effects on Diesel engine combustion, Proc. Combust. Inst., vol. 25, pp. 175-181, 1994.

Shih, A. T. and Megaridis, C. M., Thermocapillary flow effects on convective droplet evaporation, Int. J. Heat Mass Transfer, vol. 39, pp. 247-257, 1996.

Shinjo, J. and Umemura, A., Simulation of liquid jet primary breakup: Dynamics of ligament and droplet formation, Int. J. Multiphase Flow, vol. 36, pp. 513-532, 2010.

Volume 26, Issue 6, 2016 
Shinjo, J. and Umemura, A., Detailed simulation of primary atomization mechanisms in Diesel jet sprays (isolated identification of liquid jet tip effects), Proc. Combust. Inst., vol. 33, pp. 2089-2097, 2011.

Shinjo, J. and Umemura, A., Droplet/turbulence interaction and early flame kernel development in an autoigniting realistic dense spray, Proc. Combust. Inst., vol. 34, pp. 1553-1560, 2013.

Shinjo, J., Xia, J., Ganippa, L. C., and Megaritis, A., Physics of puffing and microexplosion of emulsion fuel droplets, Phys. Fluids, vol. 26, 103302, 2014.

Shinjo, J., Xia, J., and Umemura, A., Droplet/ligament modulation of local small-scale turbulence and scalar mixing in a dense fuel spray, Proc. Combust. Inst., vol. 35, pp. 1595-1602, 2015.

Sirignano, W. A., Fuel droplet vaporization and spray combustion theory, Prog. Energy Combust. Sci., vol. 9, pp. 291-322, 1983.

Sirignano, W. A., Fluid Dynamics and Transport of Droplets and Sprays, Cambridge: Cambridge University Press, 2010.

Sussman, M., Smereka, P., and Osher, S., A level set approach for computing solutions to incompressible two-phase flow, J. Comput. Phys., vol. 114, pp. 146-159, 1994.

Sussman, M. and Puckett, E. G., A coupled level set and volume-of-fluid method for computing 3D and axisymmetric incompressible two-phase flows, J. Comput. Phys., vol. 162, pp. 301$337,2000$.

Suzuki, Y., Harada, T., Watanabe, H., Shoji, M., Matsushita, Y., Aoki, H., and Miura, T., Visualization of aggregation process of dispersed water droplets and the effect of aggregation on secondary atomization of emulsified fuel droplets, Proc. Combust. Inst., vol. 33, pp. 20632070, 2011.

Takewaki, H., Nishiguchi, A., and Yabe, T., Cubic interpolated pseudo-particle method (CIP) for solving hyperbolic-type equations, J. Comput. Phys., vol. 61, pp. 261-268, 1985.

Talley, D. G. and Yao, S. C., A semi-empirical approach to thermal and composition transients inside vaporizing fuel droplets, Proc. Combust. Inst., vol. 21, pp. 609-616, 1986.

Tanguy, S., Ménard, T., and Berlemont, A., A level set method for vaporizing two-phase flows, J. Comput. Phys., vol. 221, pp. 837-853, 2007.

Tarlet, D., Bellettre, J., Tazerout, M., and Rahmouni, C., Prediction of micro-explosion delay of emulsified fuel droplets, Int. J. Therm. Sci., vol. 48, pp. 449-460, 2009.

Tong, A. Y. and Sirignano, W. A., Analytical solution for diffusion and circulation in a vaporizing droplet, Proc. Combust. Inst., vol. 19, pp. 1007-1020, 1982.

Vega, C. and de Miguel, E., Surface tension of the most popular models of water by using the test-area simulation method, J. Chem. Phys., vol. 126, 154707, 2007.

Watanabe, H. and Okazaki, K., Visualization of secondary atomization in emulsified-fuel spray flow by shadow imaging, Proc. Combust. Inst., vol. 34, pp. 1651-1658, 2013.

Watanabe, H., Matsushita, Y., Aoki, H., and Miura, T., Numerical simulation of emulsified fuel spray combustion with puffing and micro-explosion, Combust. Flame, vol. 157, pp. 839-852, 2010.

Xia, J. and Luo, K. H., Direct numerical simulation of diluted combustion by evaporating droplets, Proc. Combust. Inst., vol. 32, pp. 2267-2274, 2009. 
Xia, J. and Luo, K. H., Direct numerical simulation of inert droplet effects on scalar dissipation rate in turbulent reacting and non-reacting shear layers, Flow, Turbul. Combust., vol. 84, pp. 397-422, 2010.

Xia, J., Zhao, H., Megaritis, A., Luo, K. H., Cairns, A., and Ganippa, L. C., Inert-droplet and combustion effects on turbulence in a diluted diffusion flame, Combust. Flame, vol. 160, pp. 366-383, 2013.

Yabe, T., Xiao, F., and Utsumi, T., The constrained interpolation profile method for multiphase analysis, J. Comput. Phys., vol. 169, pp. 556-593, 2001.

Young, N. O., Goldstein, J. S., and Block, M. J., The motion of bubbles in a vertical temperature gradient, J. Fluid Mech., vol. 6, pp. 350-356, 1959.

Zeng, Y. and Lee, C. F., Modeling droplet breakup processes under micro-explosion conditions, Proc. Combust. Inst., vol. 31, pp. 2185-2193, 2007. 